\title{
On fake lens spaces with fundamental group of order a power of 2
}

\author{
TIBOR MACKO \\ CHRISTIAN WEGNER
}

\begin{abstract}
We present a classification of fake lens spaces of dimension $\geq 5$ which have as fundamental group the cyclic group of order $N=2^{K}$, which extends the results of Wall and others in the case $N=2$.
\end{abstract}

$57 \mathrm{R} 65,57 \mathrm{~S} 25$

\section{Introduction}

A fake lens space is the orbit space of a free action of a finite cyclic group $G$ on a sphere $S^{2 d-1}$. It is a generalization of the notion of a lens space which is the orbit space of a free action which comes from a unitary representation. The classification of lens spaces is a classical topic in algebraic topology and algebraic $K$-theory well explained for example in Milnor [8]. For the classification of fake lens spaces in dimension $\geq 5$ methods of surgery theory are especially suitable. The classification of fake lens spaces with $G$ of order $N=2$ or $N$ odd was obtained and published in the books Wall [13] and López de Medrano [7]. Since then, the problem has remained open for $N \neq 2$ even. In this paper we address the classification for $N=2^{K}$.

One reason why the classification for all $N$ was not finished in Wall [13] seems to be that the so-called $L$-groups $L_{n}^{s}(G)$ for $G=\mathbb{Z}_{N}$ were unknown for $N$ even. This is not the case anymore; see for example Hambleton and Taylor [3]. Using this additional information and the general methods of Wall from [13, Chapter 14] we reduce the classification question to a problem in the representation theory of $G$. The main contribution of the present paper is that we develop calculational methods for solving this rather complicated problem and we obtain the solution for $N=2^{K}$. In the follow-up paper [5] we complete the analysis for all $N \in \mathbb{N}$.

The classification of fake lens spaces up to simple homotopy equivalence for all $N \in \mathbb{N}$ via Reidemeister torsion is described in [13, Chapter 14E]. The desired homeomorphism classification within a simple homotopy type can be formulated in terms of the simple structure set $\mathcal{S}^{s}(X)$ of a closed topological $n$-manifold $X$. An element of $\mathcal{S}^{s}(X)$ is 
represented by a simple homotopy equivalence $f: M \rightarrow X$ from a closed topological $n$-manifold $M$. Two such $f: M \rightarrow X, f^{\prime}: M^{\prime} \rightarrow X$ are equivalent if there exists a homeomorphism $h: M \rightarrow M^{\prime}$ such that $f^{\prime} \circ h \simeq f$. The simple structure set $\mathcal{S}^{s}(X)$ is a priori just a pointed set with the base point id: $X \rightarrow X$. However, it can also be endowed with a preferred structure (in some sense) of an abelian group (see Ranicki [12, Chapter 18]). There exist versions of the simple structure set for smooth and PL-manifolds, however, in this paper we only deal with the topological simple structure set.

In general the simple structure set of an $n$-manifold for $n \geq 5$ can be determined by examining the surgery exact sequence which is recalled below as (3-1). Besides determining $\mathcal{S}^{s}(X)$ it is also important to find invariants that distinguish its elements. In fact the calculation of $\mathcal{S}^{S}(X)$ is often conducted by combining the surgery exact sequence with such invariants. This is the case also for fake lens spaces. Here it follows from the calculations of Wall in [13, Chapter 14E] that the simple structure set is detected by the $\rho$-invariant of Atiyah and Singer [1] and Wall [13, Chapter 14B], and by the so-called normal invariants. Our main Theorem 1.2 calculates the simple structure set explicitly when $N=2^{K}$. This should be seen as an improvement of the detecting result of Wall. This interpretation follows from Corollary 1.3 which says that if $N=2^{K}$ there is another collection of invariants which yields a oneto-one correspondence. The collection contains the $\rho$-invariant as before, but the other invariants are new. They depend on a certain choice and certainly a geometric interpretation would still be desirable.

Another issue that arises is the action of the group of simple homotopy equivalences $G^{s}(X)$ of a manifold $X$ on $\mathcal{S}^{s}(X)$ by postcomposition. The orbits of this action are the homeomorphism types of manifolds simple homotopy equivalent to $X$ rather than homeomorphism types of manifolds equipped with a simple homotopy equivalence to $X$. Hence it is an interesting question to describe the action. Following Wall one can slightly modify the question and study the polarized homeomorphism types of polarized fake lens spaces. These are fake lens spaces equipped with a choice of orientation and a choice of a generator of the fundamental group. Corollary 1.4 describes this classification.

Both authors are supported by SFB 478 Geometrische Strukturen in der Mathematik, Münster.

\section{Statement of results}

Definition 1.1 A fake lens space $L^{2 d-1}(\alpha)$ is a manifold obtained as the orbit space of a free action $\alpha$ of the group $G=\mathbb{Z}_{N}$ on $S^{2 d-1}$. 
The fake lens space $L^{2 d-1}(\alpha)$ is a $(2 d-1)$-dimensional manifold with fundamental group $\pi_{1}\left(L^{2 d-1}(\alpha)\right) \cong G=\mathbb{Z}_{N}$ and universal cover $S^{2 d-1}$. The main theorem in this paper is:

Theorem 1.2 Let $L^{2 d-1}(\alpha)$ be a fake lens space with $\pi_{1}\left(L^{2 d-1}(\alpha)\right) \cong \mathbb{Z}_{N}$ where $N=2^{K}$ and $d \geq 3$. Then we have

$$
\mathcal{S}^{s}\left(L^{2 d-1}(\alpha)\right) \cong \bar{\Sigma} \oplus \bar{T} \cong \bar{\Sigma} \oplus \bigoplus_{i=1}^{c} \mathbb{Z}_{2} \oplus \bigoplus_{i=1}^{c} \mathbb{Z}_{2^{\min \{K, 2 i\}}}
$$

where $\bar{\Sigma}$ is a free abelian group of rank $N / 2-1$ if $d=2 e+1$ and $N / 2$ if $d=2 e$ and $c=\lfloor(d-1) / 2\rfloor$.

The isomorphism (1-1) has an interpretation in terms of known geometric invariants. These are the reduced $\rho$-invariant and the normal invariants from surgery theory as follows.

The reduced $\rho$-invariant is a homomorphism

$$
\tilde{\rho}: \mathcal{S}^{s}\left(L^{2 d-1}(\alpha)\right) \longrightarrow \mathbb{Q} R \widehat{G}^{(-1)^{d}}
$$

where the target is the underlying abelian group of the $(-1)^{d}$-eigenspace of the rationalized complex representation ring of $G$ modulo the ideal generated by the regular representation. The group $\bar{\Sigma}$ is defined as the image of $\tilde{\rho}$.

The normal invariant is a homomorphism $\eta: \mathcal{S}^{s}\left(L^{2 d-1}(\alpha)\right) \rightarrow \mathcal{N}\left(L^{2 d-1}(\alpha)\right)$ with the target the group of normal invariants from surgery theory, which is easily calculable. The reduced $\rho$-invariant induces the homomorphism

$$
[\widetilde{\rho}]: \widetilde{\mathcal{N}}\left(L^{2 d-1}(\alpha)\right) \longrightarrow \mathbb{Q} R_{\widehat{G}}{ }^{(-1)^{d}} / 4 \cdot R_{\widehat{G}^{(-1)^{d}}} .
$$

Here the source is the subgroup of $\mathcal{N}\left(L^{2 d-1}(\alpha)\right)$ given by the image of $\eta$ and in the target we have the quotient group modulo the subgroup of elements in the $(-1)^{d}-$ eigenspace of the representation ring which are divisible by 4 . We use formulas of Wall to show relations between the invariants $\tilde{\rho}$ and $\eta$ in Proposition 4.12.

The group $\bar{T}$ is defined as the kernel of $[\widetilde{\rho}$. In the proof of Theorem 5.1 we describe a map $\lambda: \bar{T} \rightarrow \mathcal{S}^{s}\left(L^{2 d-1}(\alpha)\right)$ which fits into a short exact sequence

$$
0 \longrightarrow \bar{T} \stackrel{\lambda}{\rightarrow} \mathcal{S}^{s}\left(L^{2 d-1}(\alpha)\right) \stackrel{\tilde{\rho}}{\rightarrow} \bar{\Sigma} \longrightarrow 0 .
$$

Since $\bar{\Sigma}$ is a free abelian group the sequence splits and we obtain the isomorphism of Theorem 1.2. We denote the projection map on $\bar{T}$ by $\mathbf{r}: \mathcal{S}^{s}\left(L^{2 d-1}(\alpha)\right) \rightarrow \bar{T}$. Our main technical result is the calculation of $\bar{T}$ in Theorems 5.2 and 5.3. It tells us that it 
is a direct sum of copies of 2-primary cyclic groups which are indexed by $1 \leq i \leq 2 c$. We denote the projection on the $i$-th summand by $\mathbf{r}_{2 i}$. Putting these considerations together we obtain the following corollary.

Corollary 1.3 Let $L^{2 d-1}(\alpha)$ be a fake lens space with $\pi_{1}\left(L^{2 d-1}(\alpha)\right) \cong \mathbb{Z}_{N}$ where $N=2^{K}$ and $d \geq 3$. There exists a collection of invariants

$$
\mathbf{r}_{4 i}: \mathcal{S}^{s}\left(L^{2 d-1}(\alpha)\right) \longrightarrow \mathbb{Z}_{2^{\min \{K, 2 i\}}} \quad \text { and } \quad \mathbf{r}_{4 i-2}: \mathcal{S}^{s}\left(L^{2 d-1}(\alpha)\right) \longrightarrow \mathbb{Z}_{2}
$$

where $1 \leq i \leq c=\lfloor(d-1) / 2\rfloor$ which together with the $\tilde{\rho}$-invariant induce a one-to-one correspondence between elements $a \in \mathcal{S}^{s}\left(L^{2 d-1}(\alpha)\right)$ and

(1) $\tilde{\rho}(a) \in \bar{\Sigma} \subset \mathbb{Q} R_{\widehat{G}^{(-1)^{d}}}$,

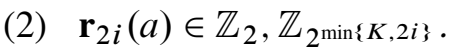

The invariants $\mathbf{r}_{4 i-2}$ are the normal invariants $\mathbf{t}_{4 i-2}$ from [13, Chapter 14E] and the invariants $\mathbf{r}_{4 i}$ are related to the invariants $\mathbf{t}_{4 i}$ from [13, Chapter 14E], but they are not the same. The invariants $\mathbf{t}_{2 i}$ can in principle be calculated using characteristic classes (see Morgan and Sullivan [9]) and for the lens spaces this has been done by Young [14], but the calculation does not include fake lens spaces. Admittedly, a similar "calculational" description of the invariants $\mathbf{r}_{4 i}$ would be desirable. In the follow-up paper [5] we offer an alternative, geometric obstruction theoretic description of the invariants $\mathbf{r}_{4 i}$ obtained via the analysis of the question which fake lens spaces can be "desuspended".

The above results are about classification within a simple homotopy type. As stated in the introduction the simple homotopy types of fake lens spaces can be distinguished by the Reidemeister torsion which is a unit in $\mathbb{Q} R_{G}$, the rational group ring of $G$ modulo the ideal generated by the norm element.

To obtain classification of fake lens spaces rather than classification of elements of the simple structure set we follow Wall and work with polarized fake lens spaces; see Definition 2.2. The simple homotopy type of a polarized lens space is given uniquely by its Reidemeister torsion as described in Proposition 2.3. That means that for two polarized fake lens spaces $L^{2 d-1}(\alpha)$ and $L^{2 d-1}(\beta)$ with the fundamental group $G$ there is a simple homotopy equivalence $f_{\alpha, \beta}: L^{2 d-1}(\alpha) \rightarrow L^{2 d-1}(\beta)$ of polarized fake lens spaces unique up to homotopy if and only if the Reidemeister torsions of $L^{2 d-1}(\alpha)$ and $L^{2 d-1}(\beta)$ coincide. This $f_{\alpha, \beta}$ gives us an element of the simple structure set $\mathcal{S}^{s}\left(L^{2 d-1}(\beta)\right)$. We can formulate the classification as follows:

Corollary 1.4 Let $L^{2 d-1}(\alpha)$ and $L^{2 d-1}(\beta)$ be polarized lens spaces with the fundamental group $G=\mathbb{Z}_{N}$, where $N=2^{K}$ and $d \geq 3$. There exists a polarized homeomorphism between $L^{2 d-1}(\alpha)$ and $L^{2 d-1}(\beta)$ if and only if 
(1) $\Delta\left(L^{2 d-1}(\alpha)\right)=\Delta\left(L^{2 d-1}(\beta)\right)$,

(2) $\rho\left(L^{2 d-1}(\alpha)\right)=\rho\left(L^{2 d-1}(\beta)\right)$,

(3) $\mathbf{r}_{2 i}\left(f_{\alpha, \beta}\right)=0$ for all $i$.

The paper is organized as follows. In Section 2 we briefly recall the simple homotopy classification of fake lens spaces. In Section 3 we recall the general machinery of surgery theory and we describe the known terms in the surgery exact sequence of the fake lens spaces. In Section 4 we recall the definition and properties of the $\rho$-invariant. Finally, in Section 5 we prove our main technical result which is the calculation of the group $\bar{T}$. Sections 2, 3 and most of Section 4 are a summary of known results. Our contribution is concentrated in a part of Section 4 and in the last Section 5.

We thank Diarmuid Crowley, Ian Hambleton, Andrew Ranicki and the anonymous referee for useful comments.

\section{Homotopy classification}

In this section we briefly recall without proofs the statements of the homotopy and simple homotopy classification of fake lens spaces from [13, Chapter 14E]. Apart from definitions only Corollary 2.4 is of importance for the rest of the paper.

We start by introducing some notation for lens spaces which are a special sort of fake lens spaces. Let $N \in \mathbb{N}$ and $\bar{k}=\left(k_{1}, \ldots k_{d}\right)$, where $k_{i} \in \mathbb{Z}$ are such that $\left(k_{i}, N\right)=1$. When $G=\mathbb{Z}_{N}$ define a representation $\alpha \bar{k}$ of $G$ on $\mathbb{C}^{d}$ by $\left(z_{1}, \ldots, z_{d}\right) \mapsto$ $\left(z_{1} e^{2 \pi i k_{1} / N}, \ldots, z_{n} e^{2 \pi i k_{d} / N}\right)$. Any free representation of $G$ on a $d$-dimensional complex vector space is isomorphic to some $\alpha_{\bar{k}}$. The representation $\alpha_{\bar{k}}$ induces a free action of $G$ on $S^{2 d-1}$ which we still denote $\alpha \bar{k}$.

Definition 2.1 A lens space $L^{2 d-1}(\alpha \bar{k})$ is a manifold obtained as the orbit pace of a free action $\alpha \bar{k}$ of the group $G=\mathbb{Z}_{N}$ on $S^{2 d-1}$ for some $\bar{k}=\left(k_{1}, \ldots k_{d}\right)$ as above. ${ }^{1}$

The lens space $L^{2 d-1}(\alpha \bar{k})$ is a $(2 d-1)$-dimensional manifold with $\pi_{1}\left(L^{2 d-1}(\alpha \bar{k})\right) \cong$ $\mathbb{Z}_{N}$. Its universal cover is $S^{2 d-1}$, hence $\pi_{i}\left(L^{2 d-1}(\alpha \bar{k})\right) \cong \pi_{i}\left(S^{2 d-1}\right)$ for $i \geq 2$. There exists a convenient choice of a CW-structure for $L^{2 d-1}(\alpha \bar{k})$ with one cell $e_{i}$ in each dimension $0 \leq i \leq 2 d-1$. Moreover, we have $H_{i}\left(L^{2 d-1}(\alpha \bar{k})\right) \cong \mathbb{Z}$ when $i=$ $0,2 d-1, H_{i}\left(L^{2 d-1}(\alpha \bar{k})\right) \cong \mathbb{Z}_{N}$ when $0<i<2 d-1$ is odd and $H_{i}\left(L^{2 d-1}(\alpha \bar{k})\right) \cong 0$ when $i \neq 0$ is even.

\footnotetext{
${ }^{1}$ In the notation of $[13$, Chapter $14 \mathrm{E}]$ we have $L(\alpha \bar{k})=L\left(N, k_{1}, \ldots, k_{n}\right)$.
} 
The classification of the lens spaces up to homotopy equivalence and simple homotopy equivalence is presented for example in Milnor [8]. The simple homotopy classification is stated in terms of Reidemeister torsion which is a unit in $\mathbb{Q} R_{G}$. This ring is defined as $\mathbb{Q} R_{G}=\mathbb{Q} \otimes R_{G}$ with $R_{G}=\mathbb{Z} G /\langle Z\rangle$ where $\mathbb{Z} G$ be the group ring of $G$ and $\langle Z\rangle$ is the ideal generated by the norm element $Z$ of $G$. We also suppose that a generator $T$ of $G$ is chosen. There is also an augmentation map $\varepsilon^{\prime}: R_{G} \rightarrow \mathbb{Z}_{N}$ [13, page 214]. The homotopy classification is stated in terms of a certain unit in $\mathbb{Z}_{N}$. These invariants also suffice for the homotopy and simple homotopy classification of finite CW-complexes $L$ with $\pi_{1}(L) \cong \mathbb{Z}_{N}$ and with the universal cover homotopy equivalent to $S^{2 d-1}$ of which fake lens spaces are obviously a special case. It is convenient to make the following definition.

Definition 2.2 A polarization of a CW-complex $L$ as above is a pair $(T, e)$ where $T$ is a choice of a generator of $\pi_{1}(L)$ and $e$ is a choice of a homotopy equivalence $e: \widetilde{L} \rightarrow S^{2 d-1}$.

Denote further by $L^{2 d-1}\left(\alpha_{k}\right)$ the lens space $L^{2 d-1}(\alpha \bar{k})$ with $\bar{k}=(1, \ldots, 1, k)$. By $L^{i}\left(\alpha_{1}\right)$ is denoted the $i$-skeleton of the lens space $L^{2 d-1}\left(\alpha_{1}\right)$. If $i$ is odd this is a lens space, if $i$ is even this is a CW-complex obtained by attaching an $i$-cell to the lens space of dimension $i-1$.

Proposition 2.3 [13, Theorem 14E.3] Let $L$ be a finite $C W$-complex as above polarized by $(T, e)$. Then there exists a simple homotopy equivalence

$$
h: L \longrightarrow L^{2 d-2}\left(\alpha_{1}\right) \cup_{\phi} e^{2 d-1}
$$

preserving the polarization. It is unique up to homotopy and the action of $G$. The chain complex differential on the right hand side is given by $\partial_{2 d-1} e^{2 d-1}=e_{2 d-2}(T-1) U$ for some $U \in \mathbb{Z} G$ which maps to a unit $u \in R_{G}$. Furthermore, the complex $L$ is a Poincaré complex.

(1) The polarized homotopy types of such $L$ are in one-to-one correspondence with the units in $\mathbb{Z}_{N}$. The correspondence is given by $\varepsilon^{\prime}(u) \in \mathbb{Z}_{N}$.

(2) The polarized simple homotopy types of such $L$ are in one-to-one correspondence with the units in $R_{G}$. The correspondence is given by $u \in R_{G}$.

The existence of a fake lens space in the homotopy type of such $L$ is addressed in [13, Theorem 14E.4]. Since the units $\varepsilon^{\prime}(u) \in \mathbb{Z}_{N}$ are exhausted by the lens spaces $L^{2 d-1}\left(\alpha_{k}\right)$ we obtain the following corollary.

Corollary 2.4 For any fake lens space $L^{2 d-1}(\alpha)$ there exists $k \in \mathbb{N}$ and a homotopy equivalence

$$
h: L^{2 d-1}(\alpha) \longrightarrow L^{2 d-1}\left(\alpha_{k}\right) .
$$




\section{The surgery exact sequence}

We proceed to the homeomorphism classification within a simple homotopy type. This is the standard task of surgery theory whose main tool is the surgery exact sequence computing the structure set $\mathcal{S}^{s}(X)$ for a given $n$-manifold $X$ with $n \geq 5$ :

$$
\cdots \rightarrow \mathcal{N}_{\partial}(X \times I) \stackrel{\theta}{\rightarrow} L_{n+1}^{s}(G) \stackrel{\partial}{\rightarrow} \mathcal{S}^{s}(X) \stackrel{\eta}{\rightarrow} \mathcal{N}(X) \stackrel{\theta}{\rightarrow} L_{n}^{s}(G),
$$

where $G=\pi_{1}(X)$. The other terms in the sequence are reviewed below. We note that, since $\mathcal{S}^{s}(X)$ is a priori only a pointed set, the "exactness" is to be understood as described in [13, Chapter 10] or [4, Chapter 5]. However, the sequence can also be made into an exact sequence of abelian groups by the identification with the algebraic surgery exact sequence of Ranicki as explained in [12, Chapter 18]. We will make use of this structure since it makes certain statements and proofs easier. However, our results can be also formulated without this identification, in a less neat way though.

By $\mathcal{N}(X)$ in (3-1) is denoted the set of normal invariants of $X$. An element of $\mathcal{N}(X)$ is represented by a degree one normal map $(f, b): M \rightarrow X$ which consists of a map $f: M \rightarrow X$ of oriented closed $n$-manifolds of degree 1 and a stable bundle map $b: v_{M} \rightarrow \xi$ from the stable normal bundle of $M$ to a stable topological reduction $\xi$ of the stable Spivak normal fibration of $X$. Two such degree one normal maps $(f, b): M \rightarrow X,\left(f^{\prime}, b^{\prime}\right): M^{\prime} \rightarrow X$ are equivalent in $\mathcal{N}(X)$ if there exists a degree one normal map $(F, B):\left(W, M, M^{\prime}\right) \rightarrow(X \times I, X \times 0, X \times 1)$ of manifolds with boundary which restricts on the two ends to $(f, b),\left(f^{\prime}, b^{\prime}\right)$ respectively. Again this is a priori a set, with a base point (id, id): $X \rightarrow X$. However, the Pontrjagin-Thom construction gives a bijection

$$
\mathcal{N}(X) \stackrel{\cong}{\rightarrow}[X ; \mathrm{G} / \mathrm{TOP}]
$$

where $[-,-]$ denotes the homotopy classes of maps and G/TOP is the classifying space for topological reductions of spherical fibrations. The $H$-space structure on G/TOP coming from Sullivan characteristic variety theorem [6, Chapter 4] (also called "disjoint union $H$-space structure" in [11]) makes $\mathcal{N}(X)$ into an abelian group. This $H$-space structure extends to an infinite loop space structure which expresses $\mathcal{N}(X)$ via localization in terms of familiar cohomology theories.

Theorem 3.1 [6] There are compatible homotopy equivalences

$$
\begin{aligned}
\mathrm{G} / \mathrm{TOP}_{(2)} & \simeq \Pi_{i \geq 1} K\left(\mathbb{Z}_{(2)}, 4 i\right) \times K\left(\mathbb{Z}_{2}, 4 i-2\right), \\
\mathrm{G} \mathrm{TOP}_{(\text {odd })} & \simeq \mathrm{BO}_{(\text {odd })}, \\
\mathrm{G} / \mathrm{TOP}_{(0)} & \simeq \mathrm{BO}_{(0)} \simeq \Pi_{i \geq 1} K(\mathbb{Q}, 4 i) .
\end{aligned}
$$


Corollary 3.2 For a fake lens space $L=L_{N}^{2 d-1}(\alpha)$ we have an isomorphism of abelian groups

$$
\mathcal{N}(L) \cong \bigoplus_{i \geq 1} H^{4 i}\left(L ; \mathbb{Z}_{(2)}\right) \oplus H^{4 i-2}\left(L ; \mathbb{Z}_{2}\right) \oplus \widetilde{K O}(L) \otimes \mathbb{Z}\left[\frac{1}{2}\right]
$$

where $\widetilde{K O}(L)=[L ; \mathrm{BO}]$.

Given $n \in \mathbb{Z}$ and $G$ a group there is defined an abelian group $L_{n}^{s}(G)$ [13, Chapter 5,6]. For $n=2 k$ it is the Witt group of based $(-1)^{k}$-quadratic forms over the group ring $\mathbb{Z} G$, for $n=2 k+1$ it is a certain group of automorphisms of based $(-1)^{k}$-quadratic forms over $\mathbb{Z} G$. An alternative description of [12] gives these groups uniformly for all $n$ as cobordism groups of bounded chain complexes of based $\mathbb{Z} G$-modules with an $n$-dimensional Poincaré duality. The precise definition is not that important for us. We are mainly interested in the invariants which detect these groups for $G \cong \mathbb{Z}_{N}$.

Theorem 3.3 For $G=1$ we have

$$
L_{n}^{s}(1) \cong \begin{cases}8 \cdot \mathbb{Z} & n \equiv 0(\bmod 4)(\text { signature }) \\ 0 & n \equiv 1(\bmod 4) \\ \mathbb{Z}_{2} & n \equiv 2(\bmod 4)(\text { Arf }) \\ 0 & n \equiv 3(\bmod 4)\end{cases}
$$

Here "signature" in the last column means that $L_{4 k}^{s}(1) \cong 8 \cdot \mathbb{Z}$ is given by the signature of a quadratic form over $\mathbb{Z}$, and "Arf" means that $L_{4 k+2}^{s}(1) \cong \mathbb{Z}_{2}$ is given by the Arf invariant of a quadratic form over $\mathbb{Z}_{2}$. For $G \neq 1$ functoriality gives maps $L_{n}^{s}(1) \rightarrow L_{n}^{s}(G)$ and $L_{n}^{s}(G) \rightarrow L_{n}^{s}(1)$ yielding the splitting

$$
L_{n}^{s}(G) \cong L_{n}^{s}(1) \oplus \widetilde{L}_{n}^{s}(G) .
$$

Further information about the $L$-groups of finite groups is obtained using representation theory. For a finite group $G$ complex conjugation induces an involution on the complex representation ring $R_{\mathbb{C}}(G)$. One can define $( \pm 1)$-eigenspaces. In terms of characters the $(+1)$-eigenspace corresponds to real characters, the $(-1)$-eigenspace corresponds to purely imaginary characters. We will denote

$$
R_{\mathbb{C}}^{ \pm}(G)=\left\{\chi \pm \chi^{-1} \mid \chi \in R_{\mathbb{C}}(G)\right\}
$$

Notice that by $R_{\mathbb{C}}^{-}(G)$ is the $(-1)$-eigenspace, whereas $R_{\mathbb{C}}^{+}(G)$ is a subspace of the $(+1)$-eigenspace which contains $(1+1)$, but does not contain 1 .

A nondegenerate $(-1)^{k}$-quadratic form over $\mathbb{Z} G$ can be complexified. One can take its associated nondegenerate $(-1)^{k}$-symmetric bilinear form and consider the positive 
and negative definite $\mathbb{C}$-vector subspaces. These become $G$-representations and hence can be subtracted in $R_{\mathbb{C}}(G)$. This process defines the $G$-signature homomorphism (see Wall [13, Chapter 13] or Ranicki [12, Chapter 22])

$$
\text { G-sign: } L_{2 k}^{s}(G) \rightarrow R_{\mathbb{C}}^{(-1)^{k}}(G) .
$$

Its image is $4 \cdot R_{\mathbb{C}}^{(-1)^{k}}(G)$. In case $G=\mathbb{Z}_{N}$ for $N=2^{K}$ the $L$-groups are completely calculated (see Hambleton and Taylor [3]): ${ }^{2}$

Theorem 3.4 For $G=\mathbb{Z}_{N}$ we have that

$$
\begin{aligned}
& L_{n}^{s}(G) \cong \begin{cases}4 \cdot R_{\mathbb{C}}^{(+1)}(G) & n \equiv 0(\bmod 4)(\mathrm{G}-\text { sign, purely real }), \\
0 & n \equiv 1(\bmod 4), \\
4 \cdot R_{\mathbb{C}}^{(-1)}(G) \oplus \mathbb{Z}_{2} & n \equiv 2(\bmod 4)(\mathrm{G}-\text { sign, purely imaginary, Arf }), \\
\mathbb{Z}_{2} & n \equiv 3(\bmod 4)(\text { codimension 1 Arf }),\end{cases} \\
& \tilde{L}_{2 k}^{s}(G) \cong 4 \cdot R_{\widehat{G}}{ }^{(-1)^{k}} \text { where } R_{\widehat{G}}{ }^{(-1)^{k}} \text { is } R_{\mathbb{C}}^{(-1)^{k}}(G) \text { modulo } \\
& \text { the regular representation. }
\end{aligned}
$$

Next we describe briefly the maps in (3-1). If $n=2 k$ the map $\theta$ is given by first making the degree one normal map $(f, b): M \rightarrow X k$-connected and then taking the quadratic refinement of the $(-1)^{k}$-symmetric bilinear form over $\mathbb{Z}[G]$ on the kernel of $f_{*}: H_{k}(\widetilde{M}) \rightarrow H_{k}(\tilde{X})$. The exactness at $\mathcal{N}(X)$ means that there is a degree one normal map $\left(f^{\prime}, b^{\prime}\right): M^{\prime} \rightarrow X$ with $f^{\prime}$ a homotopy equivalence in the normal cobordism class of $(f, b)$ if and only if $\theta(f, b)=0$.

The map $\eta$ is given by taking the stable normal bundle $v_{M}$ of $f: M \stackrel{\simeq_{s}}{\longrightarrow} X$ and associating to it $(f, b): M \rightarrow X$ with $b: v_{M} \rightarrow\left(f^{-1}\right)^{*} v_{M}$ induced by $f$.

To describe $\partial$ we need the realization theorem for elements of $L_{n}^{s}(G)$. It says that if $M^{n-1}$ is a manifold and $x \in L_{n}^{s}(G)$ there exists a degree one normal map $(F, B):\left(W, \partial_{0} W, \partial_{1} W\right) \rightarrow(M \times I, M \times 0, M \times 1)$, where $I=[0,1]$, such that $\partial_{0} F: \partial_{0} W \rightarrow M \times 0$ is a homeomorphism, $\partial_{1} F: \partial_{1} W \rightarrow M \times 1$ is a simple homotopy equivalence and $\theta(F, B)=x$. The "map" $\partial$ in fact means that there is an action of $L_{n}^{s}(G)$ on $\mathcal{S}^{s}(X)$ given as follows. Let $f: M \rightarrow X \in \mathcal{S}^{s}(X)$ and $x \in L_{n}^{s}(G)$, then $\partial(x, f)$ is given by $\partial_{1} F_{1} \circ f: \partial_{1} W \rightarrow X$ where $(F, B): W \rightarrow M \times I$ realizes $x$. When the abelian group structure of [12, Chapter 18] is imposed on $\mathcal{S}^{s}(X)$ the action $\partial$ corresponds to the group action of the subgroup generated by the image of $\partial$ on $\mathcal{S}^{s}(X)$.

\footnotetext{
${ }^{2}$ The choice of the notation in the last line is explained later in Section 4.
} 
Hence the problem of determining $\mathcal{S}^{S}(X)$ in general consists of determining firstly $\mathcal{N}(X)$, which is tractable via standard algebraic topology, secondly $L_{n}^{s}(G)$ which we know in our case, thirdly determining the maps $\partial, \eta, \theta$ and finally solving an extension problem which is left over.

Remark 3.5 One can also define the structure set $\mathcal{S}^{h}(X)$ of an $n$-manifold $X$. Here, in comparison with the definition of $\mathcal{S}^{S}(X)$, one replaces simple homotopy equivalences by homotopy equivalences and the homeomorphism relation by the $h-$ cobordism relation. There is a version of the sequence (3-1) in this situation and again the theory of [12, Chapter 18] makes it into a long exact sequence of abelian groups. The obvious map $\mathcal{S}^{s}(X) \rightarrow \mathcal{S}^{h}(X)$ is a homomorphism.

\subsection{Complex projective spaces}

We also need the discussion of the classification problem for the complex projective spaces. This is useful also since the discussion is simpler in this case and will give us a simple example of the strategy we will need later.

The complex projective space $\mathbb{C} P^{d-1}$ is defined as the quotient of the diagonal $S^{1}-$ action on $S^{2 d-1}=S^{1} * \cdots * S^{1}$ ( $d$-factors). As a real manifold it has dimension $2 d-2$ and $\pi_{1}\left(\mathbb{C} P^{n}\right)=1$. Hence from Theorem 3.3 we have that the surgery exact sequence for $\mathbb{C} P^{d-1}$ becomes the short exact sequence

$$
0 \rightarrow \mathcal{S}^{s}\left(\mathbb{C} P^{d-1}\right) \rightarrow \mathcal{N}\left(\mathbb{C} P^{d-1}\right) \stackrel{\theta}{\rightarrow} L_{2 d-2}^{s}(1) \rightarrow 0 .
$$

For the normal invariants we have

$$
\mathcal{N}\left(\mathbb{C} P^{d-1}\right) \cong \bigoplus_{i=1}^{\lfloor(d-1) / 2\rfloor} H^{4 i}\left(\mathbb{C} P^{d-1} ; \mathbb{Z}\right) \oplus \bigoplus_{i=1}^{\lfloor d / 2\rfloor} H^{4 i-2}\left(\mathbb{C} P^{d-1} ; \mathbb{Z}_{2}\right)
$$

Further we can identify the factors

$$
\begin{aligned}
\mathbf{s}_{4 i}: \mathcal{N}\left(\mathbb{C} P^{d-1}\right) & \rightarrow H^{4 i}\left(\mathbb{C} P^{d-1} ; \mathbb{Z}\right) \cong \mathbb{Z} \cong L_{4 i}(1) \\
\mathbf{s}_{4 i-2}: \mathcal{N}\left(\mathbb{C} P^{d-1}\right) & \rightarrow H^{4 i-2}\left(\mathbb{C} P^{d-1} ; \mathbb{Z}_{2}\right) \cong \mathbb{Z}_{2} \cong L_{4 i-2}(1)
\end{aligned}
$$

as surgery obstructions of degree one normal maps obtained from $(f, b): M \rightarrow \mathbb{C} P^{d-1}$ by first making $f$ transverse to $\mathbb{C} P^{k-1}$ (for $s_{2 i}$ where $i=k-1$ ) and then taking the surgery obstruction of the degree one map obtained by restricting to the preimage of $\mathbb{C} P^{i}$. The maps $\mathbf{s}_{2 i}$ are called the splitting invariants. We will sometimes use (3-5) to identify the elements of $\mathcal{N}\left(\mathbb{C} P^{d-1}\right)$ by $s=\left(s_{2 i}\right)_{i}$. 
The surgery obstruction map $\theta$ takes the top summand of $\mathcal{N}\left(\mathbb{C} P^{d-1}\right)$ isomorphically onto $L_{2 d-2}^{s}(1)$. Hence the short exact sequence (3-4) splits and we obtain the bijection of $\mathcal{S}^{s}\left(\mathbb{C} P^{d-1}\right)$ given by the splitting invariants $\mathbf{s}_{2 i}$ for $0<i<d-1$ :

$$
\bigoplus_{0<i<d-1} \mathbf{s}_{2 i}: \mathcal{S}^{s}\left(\mathbb{C} P^{d-1}\right) \stackrel{\cong}{\rightarrow} \bigoplus_{0<i<d-1} L_{2 i}^{s}(1)
$$

If we think of $\mathcal{S}^{s}\left(\mathbb{C} P^{d-1}\right)$ as of an abelian group via Ranicki's identification [12, Chapter 18], then the map (3-8) is an isomorphism.

\subsection{Preliminaries for lens spaces}

When $X$ is a fake lens space $L^{2 d-1}(\alpha)$ with $\pi_{1}\left(L^{2 d-1}(\alpha)\right) \cong G=\mathbb{Z}_{N}$ for $N=2^{K}$ we obtain some information about the surgery exact sequence for $L^{2 d-1}(\alpha)$ from Corollary 3.2 and Theorem 3.4. In more detail,

(3-9) $\mathcal{N}\left(L^{2 d-1}(\alpha)\right) \cong \bigoplus_{i=1}^{\lfloor(d-1) / 2\rfloor} H^{4 i}\left(L^{2 d-1}(\alpha) ; \mathbb{Z}\right) \oplus \bigoplus_{i=1}^{\lfloor d / 2\rfloor} H^{4 i-2}\left(L^{2 d-1}(\alpha) ; \mathbb{Z}_{2}\right)$

We denote the factors

$$
\begin{gathered}
\mathbf{t}_{4 i}: \mathcal{N}\left(L^{2 d-1}(\alpha)\right) \rightarrow H^{4 i}\left(L^{2 d-1}(\alpha) ; \mathbb{Z}\right) \cong \mathbb{Z}_{2^{K}} \\
\mathbf{t}_{4 i-2}: \mathcal{N}\left(L^{2 d-1}(\alpha)\right) \rightarrow H^{4 i-2}\left(L^{2 d-1}(\alpha) ; \mathbb{Z}_{2}\right) \cong \mathbb{Z}_{2}
\end{gathered}
$$

and similarly as above we will sometimes use (3-9) to identify the elements of $\mathcal{N}\left(L^{2 d-1}(\alpha)\right)$ by $t=\left(t_{2 i}\right)_{i}$. More information is obtained from the following:

\section{Theorem 3.6 [13]}

(1) If $d=2 e$, then the map

$$
\theta: \mathcal{N}\left(L^{2 d-1}(\alpha)\right) \rightarrow L_{2 d-1}^{s}(G)=L_{4 e-1}^{s}(G)=\mathbb{Z}_{2}
$$

is given by $\theta(x)=\mathbf{t}_{4 e-2}(x) \in \mathbb{Z}_{2}$.

(2) The map

$$
\theta: \mathcal{N}_{\partial}\left(L^{2 d-1}(\alpha) \times I\right) \rightarrow L_{2 d}^{s}(G)
$$

maps onto the summand $L_{2 d}^{s}(1)$.

Hence we obtain the short exact sequence

$$
0 \rightarrow \widetilde{L}_{2 d}^{s}(G) \stackrel{\partial}{\rightarrow} \mathcal{S}^{s}\left(L^{2 d-1}(\alpha)\right) \stackrel{\eta}{\rightarrow} \widetilde{\mathcal{N}}\left(L^{2 d-1}(\alpha)\right) \rightarrow 0
$$


where

$$
\begin{aligned}
& \widetilde{\mathcal{N}}\left(L^{4 e-1}(\alpha)\right)=\operatorname{ker}\left(\mathbf{t}_{4 e-2}: \mathcal{N}\left(L^{4 e-1}(\alpha)\right) \rightarrow H^{4 e-2}\left(L^{4 e-1}(\alpha) ; \mathbb{Z}_{2}\right) \cong \mathbb{Z}_{2}\right), \\
& \widetilde{\mathcal{N}}\left(L^{4 e+1}(\alpha)\right)=\mathcal{N}\left(L^{4 e+1}(\alpha)\right) .
\end{aligned}
$$

In other words

$$
\widetilde{\mathcal{N}}\left(L^{2 d-1}(\alpha)\right) \cong \bigoplus_{i=1}^{c} \mathbb{Z}_{N} \oplus \bigoplus_{i=1}^{c} \mathbb{Z}_{2}
$$

where $c=\lfloor(d-1) / 2\rfloor$. The first term in the sequence (3-12) is understood by Theorem 3.4 , the third term is understood by (3-13). Hence we are left with an extension problem.

\subsection{The join construction}

We will make use of the join construction from [13, Chapter 14A]. It can be explained as follows. Let $G$ be a group (in our case $G \leq S^{1}$ ) acting freely on the spheres $S^{m}$ and $S^{n}$. Then the two actions extend to the join $S^{m+n+1} \cong S^{m} * S^{n}$ and the resulting action remains free. When we are given two lens spaces (complex projective spaces) $L$ and $L^{\prime}$, we can pass to universal covers ( $S^{1}$-bundles), form the join and then pass to the quotient again. The resulting space is again a fake lens space (a fake complex projective space). This operation will be denoted $L * L^{\prime}$ and it will be called the join. When $L^{\prime}=L^{1}\left(\alpha_{1}\right)$ we call this operation a suspension.

The join with $L^{1}\left(\alpha_{k}\right)$ defines a map $\Sigma_{k}: \mathcal{S}^{s}\left(L^{2 d-1}\left(\alpha_{1}\right)\right) \longrightarrow \mathcal{S}^{s}\left(L^{2 d+1}\left(\alpha_{k}\right)\right)$. The inclusion $L^{2 d-1}\left(\alpha_{1}\right) \subset L^{2 d+1}\left(\alpha_{k}\right)$ induces a restriction map on the normal invariants res: $\mathcal{N}\left(L^{2 d+1}\left(\alpha_{k}\right)\right) \longrightarrow \mathcal{N}\left(L^{2 d-1}\left(\alpha_{1}\right)\right)$ and we have a commutative diagram [13, Lemma 14A.3]:

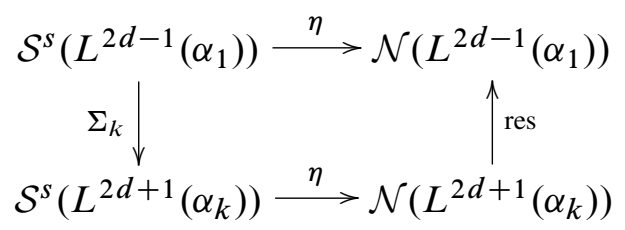

Note that we have $t_{2 i}=\operatorname{res}\left(t_{2 i}\right)$. Hence the map

$$
\text { res: } \widetilde{\mathcal{N}}\left(L^{2 d+1}\left(\alpha_{1}\right)\right) \longrightarrow \widetilde{\mathcal{N}}\left(L^{2 d-1}\left(\alpha_{1}\right)\right)
$$

is an isomorphism when $d=2 e+1$ and it is onto when $d=2 e$ with the kernel equal to $\mathbb{Z}_{N}\left(t_{4 e}\right)$. A similar diagram exists for the situation $\mathbb{C} P^{d}=\mathbb{C} P^{d-1} * \mathrm{pt}$. 
The map $\Sigma_{k}$ is a homomorphism when the structure sets are equipped with the abelian groups structure from [12, Chapter 18]. To see this notice that

$$
L^{2 d+1}\left(\alpha_{k}\right)=E(v) \cup_{S(v)} C
$$

where $E(v)$ is the total space of the normal disk-bundle of $L^{2 d-1}\left(\alpha_{1}\right)$ in $L^{2 d+1}\left(\alpha_{k}\right)$, $S(v)$ is the associated sphere-bundle and $C$ is the complement (it is the total space of a disk-bundle over $L^{1}\left(\alpha_{k}\right)$ ). Then there is a commutative diagram

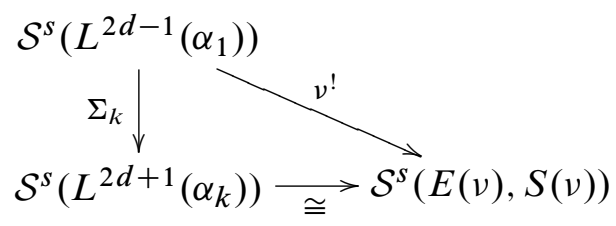

The map in the bottom row is obtained using [13, Theorem 12.1]. It follows from the calculation $\mathcal{S}_{\partial}^{S}(C)=0$ that it is an isomorphism. The map $v^{!}$is the transfer map obtained via pullback. This coincides with the algebraic surgery transfer map from [12, Chapter 21]. ${ }^{3}$

\section{The $\rho$-invariant}

We review the definition of the $\rho$-invariant for odd-dimensional manifolds and some of its properties from Atiyah and Singer [1] and Wall [13]. It will provide us with a map from the short exact sequence (3-12) to a certain short exact sequence coming from representation theory of $G$. Studying this map will enable us to solve the extension problem we are left with in the next section.

\subsection{Definitions}

Let $G$ be a compact Lie group acting smoothly on a smooth manifold $Y^{2 d}$. The middle intersection form becomes a nondegenerate $(-1)^{d}$-symmetric bilinear form on which $G$ acts. As explained earlier, such a form yields an element in the representation ring $R(G)$ which we denote by $\mathrm{G}-\operatorname{sign}(Y)$. The discussion in Section 3 also tells us that we have $\mathrm{G}-\operatorname{sign}(Y) \in R^{(-1)^{d}}(G)$ which in terms of characters means that we obtain a real (purely imaginary) character, which will be denoted as

$$
\mathrm{G}-\operatorname{sign}(-, Y): g \in G \mapsto \mathrm{G}-\operatorname{sign}(g, Y) \in \mathbb{C} .
$$

\footnotetext{
${ }^{3}$ We thank A Ranicki for informing us about the last claim.
} 
The (cohomological version of the) Atiyah-Singer $G$-index theorem [1, Theorem (6.12)] tells us that if $Y$ is closed then for all $g \in G$

$$
\mathrm{G}-\operatorname{sign}(g, Y)=L(g, Y) \in \mathbb{C},
$$

where $L(g, Y)$ is an expression obtained by evaluating certain cohomological classes on the fundamental classes of the $g$-fixed point submanifolds $Y^{g}$ of $Y$. In particular if the action is free then $\mathrm{G}-\operatorname{sign}(g, Y)=0$ if $g \neq 1$. This means that $\mathrm{G}-\operatorname{sign}(Y)$ is a multiple of the regular representation. This theorem was generalized by Wall to topological semifree actions on topological manifolds, which is the case we will need in this paper [13, Chapter 14B]. The assumption that $Y$ is closed is essential here, and motivates the definition of the $\rho$-invariant. In fact, Atiyah and Singer provide two definitions. For the first one also needs the result of Conner and Floyd [2] that for an odd-dimensional manifold $X$ with a finite fundamental group there always exists a $k \in \mathbb{N}$ and a manifold with boundary $(Y, \partial Y)$ such that $\pi_{1}(Y) \cong \pi_{1}(X)$ and $\partial Y=k \cdot X$.

Definition 4.1 [1, Remark after Corollary 7.5] Let $X^{2 d-1}$ be a closed manifold with $\pi_{1}(X) \cong G$ a finite group. Define

$$
\rho(X)=\frac{1}{k} \cdot \mathrm{G}-\operatorname{sign}(\tilde{Y}) \in \mathbb{Q} R^{(-1)^{d}}(G) /\langle\mathrm{reg}\rangle
$$

for some $k \in \mathbb{N}$ and $(Y, \partial Y)$ such that $\pi_{1}(Y) \cong \pi_{1}(X)$ and $\partial Y=k \cdot X$. The symbol $\langle$ reg $\rangle$ denotes the ideal generated by the regular representation.

By the Atiyah-Singer $G$-index theorem [1, Theorem (6.12)] is $\rho$ well defined.

Definition 4.2 Let $G$ be a compact Lie group acting freely on a manifold $\tilde{X}^{2 d-1}$. Suppose in addition that there is a manifold with boundary $(Y, \partial Y)$ on which $G$ acts (not necessarily freely) and such that $\partial Y=\tilde{X}$. Define

$$
\rho_{G}(\tilde{X}): g \in G \mapsto \mathrm{G}-\operatorname{sign}(g, Y)-L(g, Y) \in \mathbb{C} .
$$

In this definition we think about the $\rho$-invariant as about a function $G \backslash\{1\} \rightarrow \mathbb{C}$. When both definitions apply (that means when $G$ is a finite group), then they coincide, that means $\rho(X)=\rho_{G}(\tilde{X})$.

For finite $G<S^{1}$ we will use special notation following [13, Proof of Proposition 14E.6 on page 222]. By $\widehat{G}$ is denoted the Pontrjagin dual of $G$, the group $\operatorname{Hom}_{\mathbb{Z}}\left(G, S^{1}\right)$. Recall that for a finite cyclic $G$ the representation ring $R(G)$ can be canonically identified with the group ring $\mathbb{Z} \widehat{G}$. Then we also have $\mathbb{Q} R(G)=\mathbb{Q} \otimes R(G)=$ $\mathbb{Q} \widehat{G}$. Dividing out the regular representation corresponds to dividing out the norm 
element, denoted by $Z$, hence $R(G) /\langle\operatorname{reg}\rangle=R \widehat{G}=\mathbb{Z} \widehat{G} /\langle Z\rangle$ and $\mathbb{Q} R(G) /\langle$ reg $\rangle=$ $\mathbb{Q} R \widehat{G}=\mathbb{Q} \widehat{G} /\langle Z\rangle$. Choosing a generator $\widehat{G}=\langle\chi\rangle$ gives the identifications $\mathbb{Q} R \widehat{G}=$ $\mathbb{Q}[\chi] /\left\langle 1+\chi+\cdots+\chi^{N-1}\right\rangle$ where $N$ is the order of $G$. In order to save space we also use the following notation $I\langle K\rangle=\left\langle 1+\chi+\cdots+\chi^{N-1}\right\rangle$.

Recall that $R(G)$ contains two eigenspaces $R(G)^{ \pm}$with respect to the conjugation action. In terms of the above identification of $R(G)$ and $R \widehat{G}$ with the polynomial rings we have identifications:

$$
\begin{aligned}
R \widehat{G}^{-} & =\left\langle\chi^{k}-\chi^{N-k} \mid k=1, \ldots,(N / 2)-1\right\rangle \\
& =\left\{p \in \mathbb{Z}[\chi] / I\langle K\rangle \mid p\left(\chi^{N-1}\right)=-p(\chi)\right\}, \\
R \widehat{G}^{+} & =\left\langle\chi^{k}+\chi^{N-k} \mid k=0, \ldots,(N / 2)-1\right\rangle \\
& =\left\{p \in \mathbb{Z}[\chi] / I\langle K\rangle \mid p\left(\chi^{N-1}\right)=p(\chi) \text { and } p(-1) \equiv 0 \bmod 2\right\} .
\end{aligned}
$$

\subsection{Properties}

The $\rho$-invariant is an $h$-cobordism invariant [1, Corollary 7.5]. For $X^{2 d-1}$ with $\pi_{1}(X) \cong G$ it defines a function of $\mathcal{S}^{s}(X)$ by sending $a=[h: M \longrightarrow X]$ to $\widetilde{\rho}(a)=$ $\rho(M)-\rho(X)$. If we put on $\mathcal{S}^{s}(X)$ the abelian group structure from [12, Chapter 18] it is not clear whether $\tilde{\rho}$ is a homomorphism in general. ${ }^{4}$ Still the following property holds always.

Proposition 4.3 For $X^{2 d-1}$ with $\pi_{1}(X) \cong G$ there is a commutative diagram

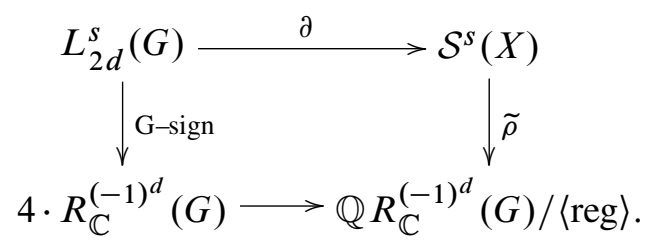

Moreover, for $z \in L_{2 d}^{s}(G)$ and $x \in \mathcal{S}^{s}(X)$ we have

$$
\tilde{\rho}(x+\partial z)=\tilde{\rho}(x)+\tilde{\rho}(\partial z) .
$$

Proof See Petrie [10, Theorem 2.3]. It essentially follows from definitions. We also use the identification of the geometrically given action of $L_{2 d}^{s}(G)$ on $\mathcal{S}^{s}(X)$ with the action coming from the abelian group structure on $\mathcal{S}^{s}(X)$ of [12, Chapter 18].

Remark 4.4 The map $\tilde{\rho}$ also obviously factors through the map $\mathcal{S}^{s}(X) \rightarrow \mathcal{S}^{h}(X)$ of Remark 3.5.

\footnotetext{
${ }^{4}$ We will see below that it is a homomorphism when $X=L^{2 d-1}(\alpha)$.
} 
When $X=L^{2 d-1}\left(\alpha_{k}\right)$, it follows from the above diagram, the exactness of the surgery exact sequence, the Atiyah-Singer $G$-index theorem and the calculation of the groups $L_{2 d}^{s}(G)$ that the action of $\widetilde{L}_{2 k}^{s}(G)$ on $\mathcal{S}^{s}\left(L^{2 k-1}\right)$ is free. In fact we have:

Proposition 4.5 There is the following commutative diagram of abelian groups and homomorphisms with exact rows

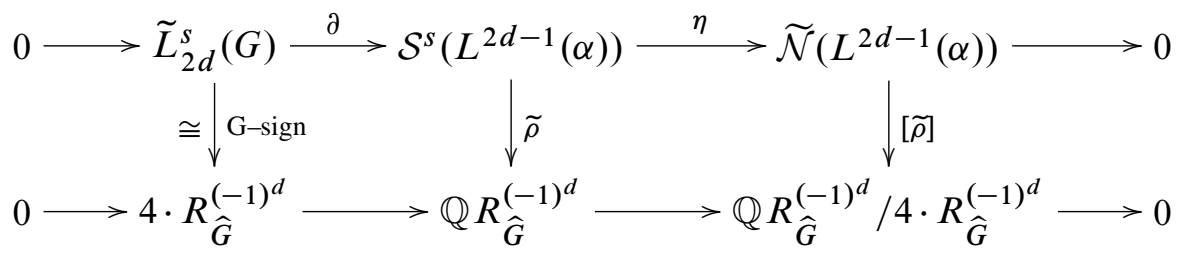

where $[\widetilde{\rho}]$ is the homomorphism induced by $\tilde{\rho}$.

All the statements follow from the previous discussion except the claim that $\tilde{\rho}$ and $[\widetilde{\rho}]$ are homomorphisms. This will be proved in this section, first for $\alpha_{1}$, then for $\alpha_{k}$, and finally for general $\alpha$. To this end we need some way to calculate the $\rho$-invariant for fake lens spaces. The formulas we obtain will give us first a good understanding of the map $[\widetilde{\rho}]$. Using certain naturality properties we will obtain also the claim about $\tilde{\rho}$.

Recall the join $L * L^{\prime}$ of the lens spaces $L$ and $L^{\prime}$ from Section 3.3. We have [13, Chapter 14A]

$$
\rho\left(L * L^{\prime}\right)=\rho(L) \cdot \rho\left(L^{\prime}\right) .
$$

For $L^{1}\left(\alpha_{k}\right)$ we have [13, Proof of Theorem 14C.4]

$$
\rho\left(L^{1}\left(\alpha_{k}\right)\right)=f_{k} \in \mathbb{Q} R_{\widehat{G}}^{\bar{G}}
$$

where $f_{k}$ is defined as follows.

Definition 4.6 For odd $k \in \mathbb{N}$ we set

$$
f_{k}:=\frac{1+\chi^{k}}{1-\chi^{k}} \quad \text { and } \quad f_{k}^{\prime}:=\frac{1-\chi+\chi^{2}-\cdots-\chi^{k-2}+\chi^{k-1}}{1+\chi+\chi^{2}+\cdots+\chi^{k-2}+\chi^{k-1}} .
$$

We abbreviate $f:=f_{1}$.

Lemma 4.7 Let $G=\mathbb{Z}_{N}$ with $N=2^{K}$. For odd $k \in \mathbb{N}$ we have

$$
f_{k} \in \mathbb{Q} R_{\bar{G}}, \quad f_{k}=f \cdot f_{k}^{\prime}, \quad f_{k}^{\prime} \in R \widehat{G} .
$$


Proof Notice that $1-\chi^{k}$ is invertible in $\mathbb{Q} R \widehat{G}$ because

$$
\left(1-\chi^{k}\right)^{-1}=-\frac{1}{N}\left(1+2 \cdot \chi^{k}+3 \cdot \chi^{2 k}+\cdots+N \cdot \chi^{(N-1) k}\right) \in \mathbb{Q} R_{\widehat{G}} .
$$

Therefore $f_{k} \in \mathbb{Q} R \widehat{G}$ and the identity

$$
\frac{1+\chi^{-k}}{1-\chi^{-k}}=-\frac{1+\chi^{k}}{1-\chi^{k}}=-f_{k}
$$

implies $f_{k} \in \mathbb{Q} R \overline{\widehat{G}}$. An easy calculation shows $f_{k}=f \cdot f_{k}^{\prime}$. That $f_{k}^{\prime} \in R_{\widehat{G}}$ follows from the fact that $1+\chi+\chi^{2}+\cdots+\chi^{k-1}$ is invertible in $R \widehat{G}$. The inverse is given by $1+\chi^{k}+\chi^{2 k}+\cdots+\chi^{(r-1) k}$ where $r$ denotes a natural number such that $r \cdot k-1$ is a multiple of $N=2^{K}$.

Also a formula of Wall which calculates the $\rho$-invariant for fake complex projective spaces will be useful. Let $a=\left[h: Q \longrightarrow \mathbb{C} P^{d-1}\right]$ be an element of $\mathcal{S}^{s}\left(\mathbb{C} P^{d-1}\right)$ and let $\tilde{h}: \widetilde{Q} \longrightarrow S^{2 d-1}$ be the associated map of $S^{1}$-manifolds. Denote $\tilde{\rho}_{S^{1}}(a):=$ $\tilde{\rho}_{S^{1}}(\widetilde{Q})-\widetilde{\rho}_{S^{1}}\left(S^{2 d-1}\right)$ defining a function of $\mathcal{S}^{s}\left(\mathbb{C} P^{d-1}\right)$.

Theorem 4.8 [13, Theorem 14C.4] Let $a=\left[h: Q \rightarrow \mathbb{C} P^{d-1}\right]$ be an element in $\mathcal{S}^{s}\left(\mathbb{C} P^{d-1}\right)$. Then for $t \in S^{1}$

$$
\tilde{\rho}_{S^{1}}(t, a)=\sum_{1 \leq i \leq\lfloor d / 2\rfloor-1} 8 \cdot \mathbf{s}_{4 i}(\eta(a)) \cdot\left(f^{d-2 i}-f^{d-2 i-2}\right) \in \mathbb{C},
$$

where $f=(1+t) /(1-t)$.

Among other things this also shows that $\tilde{\rho}_{S^{1}}$ is a homomorphism of $\mathcal{S}^{s}\left(\mathbb{C} P^{d-1}\right)$. Coming back to lens spaces recall that there is an $S^{1}$-bundle (better $L^{1}\left(\alpha_{1}\right)$-bundle) $p: L^{2 d-1}\left(\alpha_{1}\right) \longrightarrow \mathbb{C} P^{d-1}$. Via pullback it induces a commutative diagram

$$
0 \longrightarrow \mathcal{S}^{s}\left(\mathbb{C} P^{d-1}\right) \stackrel{\eta}{\longrightarrow} \mathcal{N}\left(\mathbb{C} P^{d-1}\right) \longrightarrow L_{2(d-1)}(1)
$$

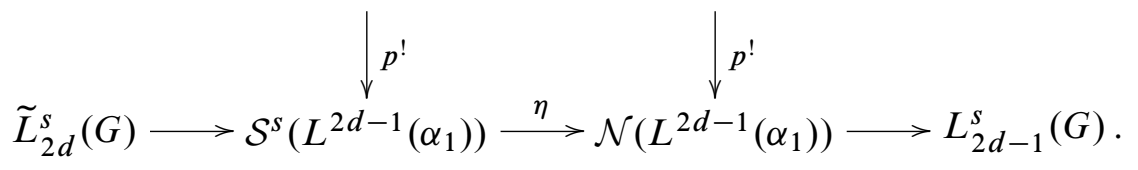

With the abelian group structure of [12, Chapter 18] the maps $p^{!}$are homomorphisms by the identification of geometric and algebraic transfers. Another way of thinking about $p^{!}$is that it is given by passing to the subgroup $G<S^{1}$. Since the $\rho$-invariant is natural for passing to subgroups we obtain: 
Corollary 4.9 [13, Theorem 14E.8] Let $a \in \mathcal{S}^{s}\left(L^{2 d-1}\left(\alpha_{1}\right)\right)$ such that $a=p^{!}(b)$ for some $b \in \mathcal{S}^{s}\left(\mathbb{C} P^{d-1}\right)$. Then

$$
\tilde{\rho}(a)=\sum_{1 \leq i \leq\lfloor d / 2\rfloor-1} 8 \cdot \mathbf{s}_{4 i}(\eta(b)) \cdot\left(f^{d-2 i}-f^{d-2 i-2}\right) \in \mathbb{Q} R \widehat{G}^{(-1)^{d}},
$$

where $f=(1+\chi) /(1-\chi)$.

The map $p^{!}: \mathcal{N}\left(\mathbb{C} P^{d-1}\right) \longrightarrow \mathcal{N}\left(L^{2 d-1}\left(\alpha_{1}\right)\right)$ is surjective, since we have

$$
p^{!}\left(s_{4 i-2}\right)=t_{4 i-2} \quad p^{!}\left(s_{4 i}\right)=t_{4 i} .
$$

By (4-5), the composition $\mathcal{S}^{s}\left(\mathbb{C} P^{d-1}\right) \rightarrow \mathcal{N}\left(\mathbb{C} P^{d-1}\right) \rightarrow \mathcal{N}\left(L^{2 d-1}\left(\alpha_{1}\right)\right)$ maps into $\widetilde{\mathcal{N}}\left(L^{2 d-1}\left(\alpha_{1}\right)\right)$. If the map $\mathcal{S}^{s}\left(\mathbb{C} P^{d-1}\right) \rightarrow \widetilde{\mathcal{N}}\left(L^{2 d-1}\left(\alpha_{1}\right)\right)$ were surjective we could use Corollary 4.9 to give a formula for the function [ $\tilde{\rho}]$. This is the case when $d=2 e$. In the case $d=2 e+1$ all the summands but the $\mathbb{Z}_{N}\left(t_{4 e}\right)$ from $\widetilde{\mathcal{N}}\left(L^{2 d-1}\left(\alpha_{1}\right)\right)$ are hit from $\mathcal{S}\left(\mathbb{C} P^{d-1}\right)$. We need the following:

Lemma 4.10 Let $d=2 e+1$ and let $a \in \mathcal{S}\left(L^{2 d-1}\left(\alpha_{1}\right)\right)$ be such that $a \mapsto \mathbf{t}(\eta(a))=$ $(0, \ldots, 1) \in \mathcal{N}\left(L^{2 d-1}\left(\alpha_{1}\right)\right)$, ie $\mathbf{t}(\eta(a))_{4 i}=0$ for $i \leq e-1$ and $\mathbf{t}(\eta(a))_{4 e}=1$. Then

$$
\tilde{\rho}(a)=8 f+z \quad \in \quad \mathbb{Q} R \overline{\widehat{G}}
$$

for some $z \in 4 \cdot R \overline{\widehat{G}}$.

Proof We will use the suspension map $\Sigma_{1}$ from Section 3.3. Our assumptions mean that $\mathbf{t}(\eta(a))$ is not in the image of the composition $\mathcal{S}\left(\mathbb{C} P^{d-1}\right) \rightarrow \mathcal{N}\left(\mathbb{C} P^{d-1}\right) \rightarrow$ $\mathcal{N}\left(L^{2 d-1}\left(\alpha_{1}\right)\right)$. However, diagram (3-14) tells us that $\mathbf{t}\left(\eta\left(\Sigma_{1}(a)\right)\right)$ is in the image of $\mathcal{S}\left(\mathbb{C} P^{(d+1)-1}\right) \rightarrow \mathcal{N}\left(\mathbb{C} P^{(d+1)-1}\right) \rightarrow \mathcal{N}\left(L^{2(d+1)-1}\left(\alpha_{1}\right)\right)$ and hence we have

$$
f \cdot \tilde{\rho}(a)+y=8 \cdot 1 \cdot\left(f^{2}-1\right) \quad \in \quad \mathbb{Q} R_{G}^{\star}
$$

for some $y \in 4 \cdot R_{G}^{*}$. We obtain the desired identity by the following calculation. Let $\hat{\rho} \in \mathbb{Q}[\chi]$ and $\hat{y} \in 4 \cdot \mathbb{Z}[\chi]$ be representatives for $\tilde{\rho}(a)$ and $y$. Then

$$
\begin{aligned}
(1+\chi)(1-\chi) \hat{\rho}+(1-\chi)^{2} \hat{y} & \equiv 8 \cdot(4 \chi) \quad \bmod I\langle K\rangle \\
(1+\chi)(1-\chi) \hat{\rho}+(1-\chi)^{2}(\hat{y}+8) & \equiv 8 \cdot(1+\chi)^{2} \quad \bmod I\langle K\rangle \\
(1+\chi)(1-\chi) \hat{\rho}+(1-\chi)^{2}(\hat{y}+8) & =8 \cdot(1+\chi)^{2}+g(\chi)\left(1+\chi+\cdots+\chi^{N-1}\right)
\end{aligned}
$$

for some $g(\chi) \in \mathbb{Q}[\chi]$. Hence $\hat{y}+8=(1+\chi) w(\chi)$ for some $w(\chi) \in \mathbb{Q}[\chi]$. Since $(\hat{y}+8) \in 4 \cdot \mathbb{Z}[\chi]$, we obtain $w(\chi) \in 4 \cdot \mathbb{Z}[\chi]$. Further write $g(\chi)=2 r+(1+\chi) g^{\prime}(\chi)=$ $r(1-\chi)+(1+\chi)\left(r+g^{\prime}(\chi)\right)$ for $r \in \mathbb{Q}, g^{\prime}(\chi) \in \mathbb{Q}[\chi]$. We have

$$
(1-\chi) \hat{\rho}+(1-\chi)^{2} w(\chi)=8 \cdot(1+\chi)+g(\chi)\left(1+\chi^{2}+\cdots \chi^{N-2}\right) \in \mathbb{Q}[\chi]
$$


and further modulo $I\langle K\rangle$

$$
\begin{aligned}
(1-\chi) \hat{\rho}+(1-\chi)^{2} w(\chi) & \equiv 8 \cdot(1+\chi)+r(1-\chi)\left(1+\chi^{2}+\cdots \chi^{N-2}\right) \\
\hat{\rho}+(1-\chi) w(\chi) & \equiv 8 \cdot f+r\left(1+\chi^{2}+\cdots \chi^{N-2}\right) .
\end{aligned}
$$

Now $(1-\chi) w(\chi)=(2-(1+\chi)) w(\chi)=2 w(\chi)-(\hat{y}+8)$. Further $2 w(\chi)=$ $w^{+}(\chi)+w^{-}(\chi)$, where $w^{ \pm}(\chi):=w(\chi) \pm w\left(\chi^{-1}\right) \in 4 \cdot \mathbb{Z}[\chi] / I\langle K\rangle$. Hence

$$
\tilde{\rho}(a)-8 \cdot f+w^{-}(\chi)=(\hat{y}+8)-w^{+}(\chi)+r\left(1+\chi^{2}+\cdots+\chi^{N-2}\right)
$$

in $\mathbb{Q}[\chi] / I\langle K\rangle$, while the left hand side of the equation lies in the $(-1)$-eigenspace and the right-hand side lies in the $(+1)$-eigenspace and hence both are equal to 0 . It follows that

$$
\tilde{\rho}(a)=8 \cdot f-w^{-}(\chi) .
$$

Putting $z=-w^{-}(\chi)$ yields the desired formula.

Lemma 4.11 Let $d=2 e+1, a \in \mathcal{S}\left(L^{2 d-1}\left(\alpha_{1}\right)\right)$ and let $\tilde{t}_{4 i} \in \mathbb{Z}$ be such that $\left[\widetilde{t}_{4 i}\right]=\mathbf{t}_{4 i}(\eta(a))$ in $\mathbb{Z}_{N}$. Then

$$
\tilde{\rho}(a)=8 \cdot \tilde{t}_{4 e} \cdot f+\sum_{1 \leq i \leq\lfloor d / 2\rfloor-1} 8 \cdot \tilde{t}_{4 i} \cdot\left(f^{d-2 i}-f^{d-2 i-2}\right)+z \quad \in \mathbb{Q} R_{\widehat{\widehat{G}}}
$$

for some $z \in 4 \cdot R \overline{\widehat{G}}$.

Proof Proof is by a straightforward modification of the proof of Lemma 4.10.

Proposition 4.12 For the map $[\widetilde{\rho}]: \widetilde{\mathcal{N}}\left(L^{2 d-1}\left(\alpha_{1}\right)\right) \longrightarrow \mathbb{Q} R_{\widehat{G}}{ }^{(-1)^{d}} / 4 \cdot R_{\widehat{G}}{ }^{(-1)^{d}}$ and an element $t=\left(t_{2 i}\right)_{i} \in \widetilde{\mathcal{N}}\left(L^{2 d-1}\left(\alpha_{1}\right)\right)$ we have that

$$
\begin{array}{ll}
\text { for } d=2 e, & {[\widetilde{\rho}](t)=\sum_{i=1}^{e-1} 8 \cdot t_{4 i} \cdot f^{d-2 i-2} \cdot\left(f^{2}-1\right)} \\
\text { for } d=2 e+1, & {[\widetilde{\rho}](t)=\sum_{i=1}^{e-1} 8 \cdot t_{4 i} \cdot f^{d-2 i-2} \cdot\left(f^{2}-1\right)+8 \cdot t_{4 e} \cdot f .}
\end{array}
$$

Remark 4.13 Strictly speaking, here and at some places below, we should replace the elements $t_{4 i} \in \mathbb{Z}_{N}$ by lifts $\tilde{t}_{4 i} \in \mathbb{Z}$, similarly as in Lemma 4.11. On the other hand, since we are passing to the quotient by $4 \cdot R \widehat{G}^{(-1)^{d}}$, the formulas become independent of the choice of the lifts. Therefore, in the interest of keeping the discussion simple, we take the liberty of thinking about $t_{4 i}$ as an element in $\mathbb{Z}$. 
Proof of Proposition 4.12 It is enough to find for each $t \in \widetilde{\mathcal{N}}\left(L^{2 d-1}\left(\alpha_{1}\right)\right)$ some $a \in \mathcal{S}^{s}\left(L^{2 d-1}\left(\alpha_{1}\right)\right)$ with $\mathbf{t}(\eta(a))=t$ and for which we can calculate $\widetilde{\rho}(a) \in \mathbb{Q} R_{\widehat{G}}{ }^{(-1)^{d}}$. If $d=2 e$ then by discussion after Corollary 4.9 there is for each normal cobordism class a fake lens space which fibers over a fake complex projective space and hence the formula from Corollary 4.9 gives the desired formula. If $d=2 e+1$ then the same reasoning applied to Lemma 4.11 gives the desired formula.

Corollary 4.14 The function $\tilde{\rho}: \mathcal{S}^{s}\left(L^{2 d-1}\left(\alpha_{1}\right)\right) \longrightarrow \mathbb{Q} R_{\widehat{G}}{ }^{(-1)^{d}}$ is a homomorphism.

Proof It is enough to show that for every $t, t^{\prime} \in \widetilde{\mathcal{N}}\left(L^{2 d-1}\left(\alpha_{1}\right)\right)$ there exist elements (not necessarily unique) $a, a^{\prime}$ in $\mathcal{S}^{s}\left(L^{2 d-1}\left(\alpha_{1}\right)\right)$ such that $\mathbf{t}(\eta(a))=t, \mathbf{t}\left(\eta\left(a^{\prime}\right)\right)=t^{\prime}$ and $\tilde{\rho}\left(a+a^{\prime}\right)=\tilde{\rho}(a)+\tilde{\rho}\left(a^{\prime}\right)$. If this holds, then for any $x, x^{\prime} \in \mathcal{S}^{s}\left(L^{2 d-1}\left(\alpha_{1}\right)\right)$ choose $a$ and $a^{\prime}$ as above corresponding to the classes $\mathbf{t}(\eta(x)), \mathbf{t}(\eta(y)) \in \mathcal{N}\left(L^{2 d-1}\left(\alpha_{1}\right)\right)$. Then $x=a+\partial(b)$ and $x^{\prime}=a^{\prime}+\partial\left(b^{\prime}\right)$ for some $b, b^{\prime} \in \partial \widetilde{L}_{2 d}^{s}(G)$ and

$$
\begin{aligned}
\widetilde{\rho}\left(x+x^{\prime}\right) & =\widetilde{\rho}\left(a+\partial b+a^{\prime}+\partial b^{\prime}\right)=\widetilde{\rho}\left(a+a^{\prime}\right)+\widetilde{\rho}\left(\partial b+\partial b^{\prime}\right) \\
& =\widetilde{\rho}(a)+\widetilde{\rho}\left(a^{\prime}\right)+\widetilde{\rho}(\partial b)+\widetilde{\rho}\left(\partial b^{\prime}\right)=\widetilde{\rho}(x)+\widetilde{\rho}\left(x^{\prime}\right) .
\end{aligned}
$$

When $d=2 e$ we can associate to a given $t \in \mathcal{N}\left(L^{2 d-1}\left(\alpha_{1}\right)\right)$ an $a \in \mathcal{S}^{s}\left(L^{2 d-1}\left(\alpha_{1}\right)\right)$ coming from the $\mathcal{S}\left(\mathbb{C} P^{d-1}\right)$, that is $a=p^{!}(b)$ where $b \in \mathcal{S}\left(\mathbb{C} P^{d-1}\right)$ such that $p^{!}(\eta(b))=t$. When $t, t^{\prime} \in \mathcal{N}\left(L^{2 d-1}\left(\alpha_{1}\right)\right)$, then $\tilde{\rho}\left(a+a^{\prime}\right)=\widetilde{\rho}\left(p^{!}(b)+p^{!}\left(b^{\prime}\right)\right)=$ $\tilde{\rho}\left(p^{!}\left(b+b^{\prime}\right)\right)=\operatorname{res}\left(\widetilde{\rho}_{S^{1}}\left(b+b^{\prime}\right)\right)=\operatorname{res}\left(\tilde{\rho}_{S^{1}}(b)+\tilde{\rho}_{S^{1}}\left(b^{\prime}\right)\right)=\operatorname{res}\left(\widetilde{\rho}_{S^{1}}(b)\right)+\operatorname{res}\left(\tilde{\rho}_{S^{1}}\left(b^{\prime}\right)\right)$ $=\tilde{\rho}(a)+\tilde{\rho}\left(a^{\prime}\right)$. Here res denotes the map on the representation rings induced by the inclusion $G<S^{1}$.

When $d=2 e+1$ and $t \in \mathcal{N}\left(L^{2 d-1}\left(\alpha_{1}\right)\right)$ we can do the same unless $t_{4 e} \neq 0$. In that case there is no fake lens space in the normal cobordism class of $\mathbf{t}$ which fibers over a fake complex projective space and we have to use a different argument. It follows from the formula in Proposition 4.12 that for $a, a^{\prime} \in \mathcal{S}^{s}\left(L^{2 d-1}\left(\alpha_{1}\right)\right)$ we have $\widetilde{\rho}\left(a+a^{\prime}\right)=\widetilde{\rho}(a)+\widetilde{\rho}\left(a^{\prime}\right)+z$ for some $z \in 4 \cdot R \widehat{G}^{-}$. Our task is to show $z=0$ for any choice of $a, a^{\prime}$. We use the fact that $\Sigma$ is a homomorphism and that we have already proved the claim for $d=2 e+2$. That implies $\left.\tilde{\rho}\left(\Sigma\left(a+a^{\prime}\right)\right)=\tilde{\rho}\left(\Sigma a+\Sigma a^{\prime}\right)\right)=$ $\tilde{\rho}(\Sigma a)+\tilde{\rho}\left(\Sigma a^{\prime}\right)=f \cdot \tilde{\rho}(a)+f \cdot \tilde{\rho}\left(a^{\prime}\right)$. On the other hand $\tilde{\rho}\left(\Sigma\left(a+a^{\prime}\right)\right)=f \cdot \tilde{\rho}\left(a+a^{\prime}\right)=$ $f \cdot \tilde{\rho}(a)+f \cdot \tilde{\rho}\left(a^{\prime}\right)+f \cdot z$. Hence it is enough to show that for any $z \in 4 \cdot R \widehat{G}^{-}$ such that $f \cdot z=0$ in $\mathbb{Q} R \widehat{G}^{+}$we have $z=0$ in $4 \cdot R \widehat{G}^{-}$. This is proved below in Lemma 5.7.

Now we proceed to the case of $\alpha_{k}$ where $k \in \mathbb{N}$ is odd. 
Proposition 4.15 For the map $[\widetilde{\rho}]: \widetilde{\mathcal{N}}\left(L^{2 d-1}\left(\alpha_{k}\right)\right) \longrightarrow \mathbb{Q} R \widehat{G}^{(-1)^{d}} / 4 \cdot R \widehat{G}^{(-1)^{d}}$ and an element $t=\left(t_{2 i}\right)_{i} \in \widetilde{\mathcal{N}}\left(L^{2 d-1}\left(\alpha_{k}\right)\right)$ we have that

$$
\begin{aligned}
& \text { for } d=2 e, {[\widetilde{\rho}](t)=\sum_{i=1}^{e-1} 8 \cdot t_{4 i} \cdot f_{k}^{\prime} \cdot f^{d-2 i-2} \cdot\left(f^{2}-1\right), } \\
& \text { for } d=2 e+1, \quad[\widetilde{\rho}](t)=\sum_{i=1}^{e-1} 8 \cdot t_{4 i} \cdot f_{k}^{\prime} \cdot f^{d-2 i-2} \cdot\left(f^{2}-1\right)+8 \cdot t_{4 e} \cdot f_{k}^{\prime} \cdot f .
\end{aligned}
$$

Proof We will use the calculation for $\alpha_{1}$ and the homeomorphisms

$$
L^{2 d+1}\left(\alpha_{k}\right) \cong L^{2 d-1}\left(\alpha_{1}\right) * L^{1}\left(\alpha_{k}\right) \quad \text { and } \quad L^{2 d+1}\left(\alpha_{k}\right) \cong L^{2 d-1}\left(\alpha_{k}\right) * L^{1}\left(\alpha_{1}\right) .
$$

For $d=2 e$ recall the diagram:

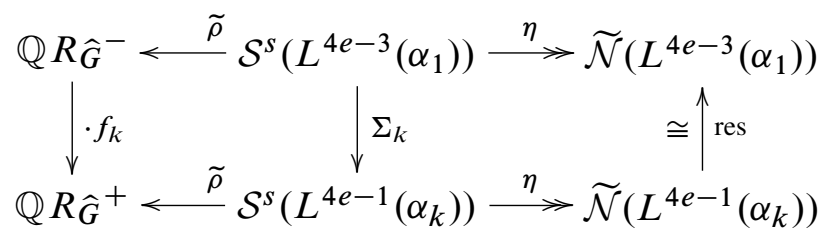

Let $t \in \widetilde{\mathcal{N}}\left(L^{4 e-1}\left(\alpha_{k}\right)\right)$. Choose $x \in \mathcal{S}^{s}\left(L^{4 e-3}\left(\alpha_{1}\right)\right)$ such that $\mathbf{t}(\eta(x))=t=\operatorname{res}(t)$. Then we have $\mathbf{t}\left(\eta\left(\Sigma_{k} x\right)\right)=t$ and $[\widetilde{\rho}]\left(\eta\left(\Sigma_{k} x\right)\right)=\left[\tilde{\rho}(x) \cdot f_{k}\right]$ can be calculated using the formulas from the case $k=1$.

For $d=2 e+1$ recall the diagram:

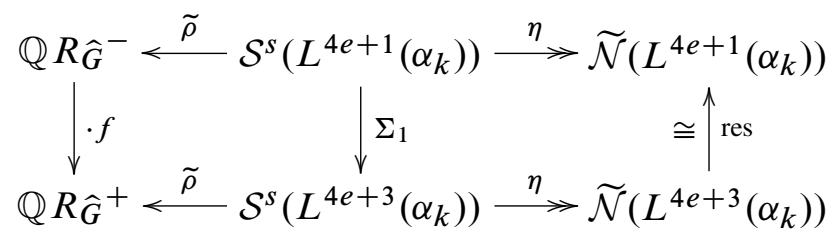

Let $t \in \widetilde{\mathcal{N}}\left(L^{4 e+1}\left(\alpha_{k}\right)\right)$. Choose $x \in \mathcal{S}^{s}\left(L^{4 e+1}\left(\alpha_{1}\right)\right)$ such that $\mathbf{t}(\eta(x))=t$. Then we have $\mathbf{t}\left(\eta\left(\Sigma_{1} x\right)\right)=t$ and $\tilde{\rho}\left(\Sigma_{1} x\right)=\tilde{\rho}(x) \cdot f$. We obtain the equation

$f \cdot \tilde{\rho}(x)+y=\sum_{i=1}^{e-1} 8 \cdot t_{4 i} \cdot f_{k}^{\prime} \cdot f^{d+1-2 i-2} \cdot\left(f^{2}-1\right)+8 \cdot t_{4 e} \cdot f_{k}^{\prime} \cdot\left(f^{2}-1\right) \in \mathbb{Q} R_{\widehat{G}^{+}}{ }^{+}$

for some $y \in 4 \cdot R \widehat{G}^{+}$using the formulas from the case $d=2 e+2$ which we have already dealt with. Now a modification of the argument from the proof of Lemma 4.10 can be used to obtain the formula for $[\widetilde{\rho}](\eta(x))$. 
Corollary 4.16 The function $\tilde{\rho}: \mathcal{S}^{s}\left(L^{2 d-1}\left(\alpha_{k}\right)\right) \longrightarrow \mathbb{Q} R \widehat{G}^{(-1)^{d}}$ is a homomorphism.

Proof Just as in the case $\alpha_{1}$ it is enough to find in each normal cobordism class an element such that the addition works for these representatives. In the case $d=2 e$ we can choose in each normal cobordism class an element coming from $\mathcal{S}^{s}\left(L^{4 e-3}\left(\alpha_{1}\right)\right)$. In the case $d=2 e+1$ there is again a problem with the summand $\mathbb{Z}_{N}\left(t_{4 e}\right)$ which can be resolved by the same reasoning as in the case $\alpha_{1}$.

Corollary 4.17 The function $\tilde{\rho}: \mathcal{S}^{s}\left(L^{2 d-1}(\alpha)\right) \longrightarrow \mathbb{Q} R \widehat{G}^{(-1)^{d}}$ is a homomorphism.

Proof From Corollary 2.4 we have that for some $k \in \mathbb{N}$ there is a homotopy equivalence $f: L^{2 d-1}(\alpha) \rightarrow L^{2 d-1}\left(\alpha_{k}\right)$. It induces a homomorphism $f_{*}: \mathcal{S}^{s}\left(L^{2 d-1}(\alpha)\right) \rightarrow$ $\mathcal{S}^{s}\left(L^{2 d-1}\left(\alpha_{k}\right)\right)$. We will show that $\tilde{\rho}=\tilde{\rho} \circ f_{*}$. This implies that $\tilde{\rho}$ is a homomorphism of $\mathcal{S}^{s}\left(L^{2 d-1}(\alpha)\right)$ since it is equal to a composition of homomorphisms.

We use the observation from Remark 4.4 and the composition formula of [11, Theorem 2.3]. Let $h: L \rightarrow L^{2 d-1}(\alpha)$ represent an element $a \in \mathcal{S}^{s}\left(L^{2 d-1}(\alpha)\right)$ and note that the homotopy equivalence $f$ represents an element in $\mathcal{S}^{h}\left(L^{2 d-1}\left(\alpha_{k}\right)\right)$, call it $b$. The composition $h \circ f$ represents another element in $\mathcal{S}^{h}\left(L^{2 d-1}\left(\alpha_{k}\right)\right)$, call it $c$. The formula of [11, Theorem 2.3] says $f_{*} a=b-c$. Now clearly

$$
\begin{aligned}
\tilde{\rho}\left(f_{*} a\right) & =\tilde{\rho}(b)-\tilde{\rho}(c) \\
& =\rho(L)-\rho\left(L^{2 d-1}\left(\alpha_{k}\right)\right)-\rho\left(L^{2 d-1}(\alpha)\right)+\rho\left(L^{2 d-1}\left(\alpha_{k}\right)\right)=\tilde{\rho}(a) .
\end{aligned}
$$

This finishes the proof.

\section{Calculations}

We want to prove Theorem 1.2 by investigating the short exact sequence (3-12) using the relation to a short exact sequence from representation theory of $G$ via the $\rho$-invariant as described in Proposition 4.5.

Notice that for any fake lens space $L^{2 d-1}(\alpha)$ there exists $k \in \mathbb{N}$ and a homotopy equivalence $h: L^{2 d-1}(\alpha) \rightarrow L^{2 d-1}\left(\alpha_{k}\right)$ (see Corollary 2.4). It induces an isomorphism $h_{*}: \mathcal{S}^{s}\left(L^{2 d-1}(\alpha)\right) \rightarrow \mathcal{S}^{s}\left(L^{2 d-1}\left(\alpha_{k}\right)\right)$. Hence it suffices to consider the case $\alpha=\alpha_{k}$.

Theorem 1.2 is obtained when we put together statements of Theorems 5.1, 5.2 and 5.3. 
Theorem 5.1 Let $\bar{T}:=\operatorname{ker}\left([\widetilde{\rho}]: \widetilde{\mathcal{N}}\left(L^{2 d-1}\left(\alpha_{k}\right)\right) \longrightarrow \mathbb{Q} R \widehat{G}^{(-1)^{d}} / 4 \cdot R \widehat{G}^{(-1)^{d}}\right)$. Then we have

$$
\mathcal{S}^{s}\left(L^{2 d-1}\left(\alpha_{k}\right)\right) \cong \bar{\Sigma} \oplus \bar{T}
$$

where $\bar{\Sigma}:=\tilde{\rho}\left(\mathcal{S}^{s}\left(L^{2 d-1}\left(\alpha_{k}\right)\right)\right)$ is a free abelian group of rank $N / 2-1$ if $d=2 e+1$ and of rank $N / 2$ if $d=2 e$.

Proof Recall the commutative diagram of Proposition 4.5. Since $\tilde{\rho}$ is a homomorphism, we have that $\bar{\Sigma}$ is a subgroup of $\mathbb{Q} R_{\widehat{G}}{ }^{(-1)^{d}}$, which as an abelian group is a direct sum of $N / 2-1$ copies of $\mathbb{Q}$ if $d=2 e+1$ and of $N / 2$ copies of $\mathbb{Q}$ if $d=2 e$. It contains a subgroup $\widetilde{\rho}\left(\partial \widetilde{L}_{2 d}^{s}(G)\right)$ which is a free abelian group of the same rank as the theorem claims for $\bar{\Sigma}$ in the respective cases. The claim about the rank of $\bar{\Sigma}$ follows.

Now replace in the diagram of Proposition 4.5 the middle and the third term of the lower sequence by the image of $\tilde{\rho}$ and by the image of $[\widetilde{\rho}]$ respectively. Then the right hand square becomes a pullback square. It follows that $\bar{T}$ is isomorphic to the kernel of the map $\tilde{\rho}$ : $\mathcal{S}^{s}\left(L^{2 d-1}\left(\alpha_{k}\right)\right) \longrightarrow \bar{\Sigma}$. We obtain a short exact sequence of abelian groups

$$
0 \longrightarrow \bar{T} \stackrel{\lambda}{\rightarrow} \mathcal{S}^{s}\left(L^{2 d-1}\left(\alpha_{k}\right)\right) \stackrel{\widetilde{\rho}}{\rightarrow} \bar{\Sigma} \longrightarrow 0
$$

where $\bar{\Sigma}$ is a free abelian group and hence the sequence splits.

So our goal is to understand the subgroup $\bar{T}$ of $\widetilde{\mathcal{N}}\left(L^{2 d-1}\left(\alpha_{k}\right)\right)$, which is a group isomorphic to the direct sum $T_{N}(d) \oplus T_{2}(d)$ of an $N$-torsion group $T_{N}(d)$ and a 2-torsion group $T_{2}(d)$

$$
T_{N}(d)=\bigoplus_{i=1}^{c} \mathbb{Z}_{N}=\bigoplus_{i=1}^{c} \mathbb{Z}_{N}\left(t_{4 i}\right) \quad T_{2}(d)=\bigoplus_{i=1}^{c} \mathbb{Z}_{2}=\bigoplus_{i=1}^{c} \mathbb{Z}_{2}\left(t_{4 i+2}\right)
$$

where $c=\lfloor(d-1) / 2\rfloor$.

Theorem 5.2 We have

$$
T_{2}(d) \subseteq \bar{T}
$$

Proof By Proposition 4.15 the formula for [ $[\widetilde{\rho}]$ only depends on $t_{4 i}$.

We will denote $\bar{T}_{N}(d):=\bar{T} \cap T_{N}(d)$ which implies $\bar{T}=\bar{T}_{N}(d) \oplus T_{2}(d)$.

Theorem 5.3 We have

$$
\bar{T}_{N}(d)=\bigoplus_{i=1}^{c} \mathbb{Z}_{2^{\min \{K, 2 i\}}}
$$

where $c=\lfloor(d-1) / 2\rfloor$. 
In view of Proposition 4.15 it is convenient to make the following reformulation. If $d=2 e$ then the group $T_{N}(d)$ can be identified with the underlying abelian group $\mathbb{Z}_{N}[x](d)$ of the truncated polynomial ring in the variable $x$ :

$$
\begin{aligned}
T_{N}(d) \stackrel{\cong}{\rightrightarrows} \mathbb{Z}_{N}[x](d):=\left\{q(x) \in \mathbb{Z}_{N}[x] \mid \operatorname{deg}(q) \leq c-1\right\} \\
t=\left(t_{4 i}\right)_{i=1}^{c} \mapsto q_{t}(x)=\sum_{i=0}^{c-1} t_{4(i+1)} \cdot x^{c-i-1} .
\end{aligned}
$$

The map $[\widetilde{\rho}]$ becomes

$$
q \mapsto 8 \cdot f_{k}^{\prime} \cdot\left(f^{2}-1\right) \cdot q\left(f^{2}\right) .
$$

If $d=2 e+1$ then the group $T_{N}(d)$ can be identified with the underlying abelian group $\mathbb{Z}_{N}[x](d)$ of the truncated polynomial ring in the variable $x$ as follows:

$$
\begin{aligned}
& T_{N}(d) \stackrel{\cong}{\rightarrow} \mathbb{Z}_{N}[x](d):=\left\{q(x) \in \mathbb{Z}_{N}[x] \mid \operatorname{deg}(q) \leq c-1\right\} \\
& t=\left(t_{4 i}\right)_{i=1}^{c} \mapsto q_{t}(x)= \sum_{i=1}^{c-1} t_{4 i} \cdot x^{c-i-1}(x-1)+t_{4 c} \\
&=\sum_{i=1}^{c-1}\left(t_{4(i+1)}-t_{4 i}\right) x^{c-i-1}+t_{4} x^{c-1}
\end{aligned}
$$

The map $[\widetilde{\rho}]$ then becomes

$$
q \mapsto 8 \cdot f_{k} \cdot q\left(f^{2}\right) .
$$

Further it is convenient to work with the underlying abelian group of

$$
\mathbb{Z}[x](d):=\{q(x) \in \mathbb{Z}[x] \mid \operatorname{deg}(q) \leq c-1\},
$$

use the formulas (5-2), (5-4) to define a map $[\hat{\rho}]: \mathbb{Z}[x](d) \longrightarrow \mathbb{Q} R_{\widehat{G}^{(-1)^{d}}}$ and study the preimage of $4 \cdot R \widehat{G}^{(-1)^{d}}$. So the task becomes to find

$$
\begin{aligned}
A_{K}^{k}(2 e) & :=\left\{q \in \mathbb{Z}[x] \mid \operatorname{deg}(q) \leq c-1,8 \cdot f_{k}^{\prime} \cdot\left(f^{2}-1\right) \cdot q\left(f^{2}\right) \in 4 \cdot \mathbb{Z}[\chi] / I\langle K\rangle\right\}, \\
A_{K}^{k}(2 e+1) & :=\left\{q \in \mathbb{Z}[x] \mid \operatorname{deg}(q) \leq c-1,8 \cdot f_{k} \cdot q\left(f^{2}\right) \in 4 \cdot \mathbb{Z}[\chi] / I\langle K\rangle\right\} .
\end{aligned}
$$

Here we have replaced $4 \cdot R \widehat{G}^{ \pm}$by the bigger ring $4 \cdot \mathbb{Z}[\chi] / I\langle K\rangle$. This is legal since when the expressions in question are in $4 \cdot \mathbb{Z}[\chi] / I\langle K\rangle$ then they always fulfill the additional conditions to be elements of $4 \cdot R \widehat{G}^{ \pm}$. We will show that $A_{K}^{k}(d)=B_{K}(d)$ where $B_{K}(d)$ is a subgroup of polynomials described in terms of certain polynomials 
$r_{n}^{ \pm}(x)$ of degree $n$ for all $n \in \mathbb{N}$. These are the best polynomial of degree $n$ in a sense that

$$
\begin{array}{r}
8 \cdot f_{k}^{\prime} \cdot\left(f^{2}-1\right) \cdot r_{n}^{+}\left(f^{2}\right) \in 4 \cdot \mathbb{Z}[\chi] / I\langle 2 n+2\rangle \\
8 \cdot f_{k} \cdot r_{n}^{-}\left(f^{2}\right) \in 4 \cdot \mathbb{Z}[\chi] / I\langle 2 n+2\rangle
\end{array}
$$

and for all polynomials $q \in \mathbb{Z}[x]$ of degree $n$ with leading coefficient 1 we have

$$
\begin{array}{r}
8 \cdot f_{k}^{\prime} \cdot\left(f^{2}-1\right) \cdot q\left(f^{2}\right) \notin 4 \cdot \mathbb{Z}[\chi] / I\langle 2 n+3\rangle \\
8 \cdot f_{k} \cdot q\left(f^{2}\right) \notin 4 \cdot \mathbb{Z}[\chi] / I\langle 2 n+3\rangle .
\end{array}
$$

We define

$$
\begin{aligned}
B_{K}(2 e): & =\left\{\sum_{n=0}^{c-1} a_{n} \cdot 2^{\max \{K-2 n-2,0\}} \cdot r_{n}^{+} \mid a_{n} \in \mathbb{Z}\right\}, \\
B_{K}(2 e+1) & :=\left\{\sum_{n=0}^{c-1} a_{n} \cdot 2^{\max \{K-2 n-2,0\}} \cdot r_{n}^{-} \mid a_{n} \in \mathbb{Z}\right\} .
\end{aligned}
$$

\section{Theorem 5.4}

$$
A_{K}^{k}(d)=B_{K}(d)
$$

Proof of Theorem 5.3 It follows from Theorem 5.4 and the definition of $B_{K}(d)$ that $A_{K}^{k}(d)$ is a free abelian subgroup of $\mathbb{Z}[x](d)$ with a basis given by polynomials $2^{\max \{K-2 n-2,0\}} \cdot r_{n}^{ \pm}$. Under the homomorphism $\mathbb{Z}[x](d) \rightarrow \mathbb{Z}_{N}[x](d)$ the subgroup $A_{K}^{k}(d)$ is mapped onto a subgroup isomorphic to a direct sum as claimed by the theorem.

Scheme of the proof of Theorem 5.4 The proof requires a formidable amount of machinery and special constructions. For better orientation we offer the following scheme. The proof is divided into five subsections:

- Section 5.1: $w_{l}$-technology

- Section 5.2: $c_{K}$-technology

- Section 5.3: Inductive properties

- Section 5.4: Good polynomials

- Section 5.5: The equality $A_{K}^{k}(d)=B_{K}(d)$

The first three subsections are preparatory, they contain the description of the tools we later need. The fourth subsection contains the definition of the polynomials $r_{n}^{-}$and 
proof of their properties. The last subsection contains the proof of the theorem, using the tools and results of the previous subsections.

For more motivation we offer the following informal discussion of main ideas. Recall that for $K=1$ the desired equation is known by [13, Chapter 14D]. Further we obviously have $A_{K}^{k}(d) \subset A_{K-1}^{k}(d)$. More interestingly, one can show that $q \in A_{K}^{k}(d)$ implies $2 \cdot q \in A_{K+1}^{k}(d)$ (Corollary 5.17). On the other hand these polynomials do not exhaust $A_{K+1}^{k}(d)$ and so the task becomes to find polynomials with the leading coefficient 1 in $A_{K+1}^{k}(d)$. However, ad-hoc calculations for low $K>1$ reveal no easy pattern for finding such polynomials.

Therefore a systematic approach is needed. At the end we proceed by induction with respect to $K$, but it turns out that for the start it is helpful to develop tools which are independent of the inductive approach. The first idea is that the ring $\mathbb{Q}[\chi] / I\langle K\rangle$ splits by the Chinese remainder theorem into a product of cyclotomic fields $\mathbb{Q}[\chi] /\left\langle 1+\chi^{2^{l}}\right\rangle$ for $0 \leq l \leq K-1$. Unfortunately, the subring $4 \cdot \mathbb{Z}[\chi] / I\langle K\rangle$ does not split into subrings of these fields. Nevertheless, the valuation in the cyclotomic field with respect to the prime ideal $(1-\chi)$ gives us useful information. We use these valuations to define functions $w_{l}$ on $\mathbb{Q}[\chi] / I\langle K\rangle$ (Definition 5.8). The functions $w_{l}$ are effectively calculable (Lemma 5.9) and we give criteria for deciding whether a $g \in \mathbb{Q}[\chi] / I\langle K\rangle$ is in $4 \cdot \mathbb{Z}[\chi] / I\langle K\rangle$ or not using $w_{l}$ (Theorem 5.11). But there is a problem, we do not obtain a necessary and sufficient condition since the criteria do not apply to all $g \in \mathbb{Q}[\chi] / I\langle K\rangle$.

Further improvement is obtained by considering the concept of a "failure" of an element $g \in \mathbb{Q}[\chi] / I\langle K\rangle$ to be in $4 \cdot \mathbb{Z}[\chi] / I\langle K\rangle$. This is the least natural number $c_{K}(g)$ such that $g \cdot(1-\chi)^{c_{K}(g)} \in 4 \cdot \mathbb{Z}[\chi] / I\langle K\rangle$ if it exists, otherwise $c_{K}(g)=\infty$. It is introduced in Section 5.2. Its usefulness stems from the fact that we know how the failure $c_{K}$ behaves for the sum of two elements in $2 \cdot \mathbb{Z}[\chi] / I\langle K\rangle$. This gives us a way to find better polynomials than those found purely by the $w_{l}$-technology. On the other hand it can also be used to show that certain linear combinations of polynomials do not lie in $4 \cdot \mathbb{Z}[\chi] / I\langle K\rangle$.

In Section 5.3 we prove Lemma 5.16 which enables induction with respect to $K$. Also the behavior of $c_{K}$ when $K$ is varied is understood (Corollary 5.17).

The $w_{l}$ and the $c_{K}$-technology are used in finding the polynomials $r_{n}^{-}$and showing their properties. This is done using a quite complicated inductive argument in Section 5.4. In the first reading this may be skipped, since what is actually essential for the proof are the properties of the polynomials $r_{n}^{-}$which are summarized in Corollary 5.27 . 
In Section 5.5 the desired equality is shown. We start with the case $d=2 e+1$, then the proof of the case $d=2 e$ is short and proceeds by a reduction to the previous case. The inclusion $B_{K}(2 e+1) \subset A_{K}^{k}(2 e+1)$ follows directly from the properties of the polynomials $r_{n}^{-}$. On the other hand, these properties together with the $c_{K}$-technology are used in proving the inclusion $A_{K}^{k}(2 e+1) \subset B_{K}(2 e+1)$, using an inductive argument with respect to $K$. Since there are inclusions $A_{K}^{k}(2 e+1) \subset A_{K-1}^{k}(2 e+1)$ and $B_{K}(2 e+1) \subset B_{K-1}(2 e+1)$ this boils down to showing that certain elements in $B_{K-1}(2 e+1)$ cannot be inside $A_{K}(2 e+1)$. Elements in $B_{K-1}(2 e+1)$ are given as linear combinations of polynomials $2^{\max \{K-2 n-3,0\}} \cdot r_{n}^{-}$, for which we can calculate the failures $c_{K}$. As indicated above the $c_{K}$-technology can now be used to show that linear combinations which are not in $B_{K}(2 e+1)$ have nonzero failure $c_{K}$ and hence cannot be inside $A_{K}^{k}(2 e+1)$.

We would still like to give more motivation for Section 5.4 which we suggest is read after the last Section 5.5. As indicated, our global strategy is to construct polynomials in $\mathbb{Z}[x](d)$ for $d=2 e+1$ which are good in a sense that they have the leading coefficient 1 and they yield elements in $4 \cdot \mathbb{Z}[\chi] / I\langle K\rangle$ for a large $K$ in comparison with the other polynomials of the same degree with leading coefficient 1 . This is what we do in Section 5.4 step-by-step. First we construct auxiliary polynomials $p_{k}$ (Definition 5.18), which are used to define "good" polynomials $q_{n}$ (Definition 5.21). These are in turn used to define "the best" polynomials $r_{n}^{-}$(Definition 5.26). This last definition is inductive, the crucial inductive step is described in Proposition 5.24. It uses a combination of the $w_{l}$-technology and the $c_{K}$-technology, whereas the definition of polynomials $p_{k}$ and $q_{n}$ only uses the $w_{l}$-technology. The "goodness" properties are summarized in Corollary $5.20\left(p_{k}\right)$, in Proposition $5.22\left(q_{n}\right)$ and in Corollary 5.27 $\left(r_{n}^{-}\right)$.

Remark 5.5 The reader may wonder that we are not using geometric constructions from Section 4 to obtain more interesting structure in the calculations. For example one could hope that varying $K$ and using the geometric transfers might be helpful. But this only yields the easy observation $A_{K}^{k}(d) \subset A_{K-1}^{k}(d)$. Also the suspension map of Section 3.3 was useful in obtaining formulas for the map [ $\tilde{\rho}]$, but is not used in the calculation. In fact here the situation is reversed, in the follow-up paper [5] we use the calculations from the present paper to understand the suspension map.

\section{$5.1 w_{l}$-technology}

For given $g \in \mathbb{Q} R \widehat{G}$ we want to decide whether $g \in 4 \cdot R \widehat{G}$ or not using the homomorphisms $\operatorname{pr}_{l}: \mathbb{Q} R_{\widehat{G}} \cong \mathbb{Q}[\chi] / I\langle K\rangle \rightarrow \mathbb{Q}[\chi] /\left\langle 1+\chi^{2^{l}}\right\rangle$ for $0 \leq l \leq K-1$. Obviously, $g \in 4 \cdot R_{\widehat{G}}$ implies $\operatorname{pr}_{l}(g) \in 4 \cdot \mathbb{Z}[\chi] /\left\langle 1+\chi^{2^{l}}\right\rangle$. Of more interest is the other direction. 
By the Chinese remainder theorem $g$ is uniquely determined by the elements $\operatorname{pr}_{l}(g)$ $(0 \leq l \leq K-1)$. More precisely, we have:

Lemma 5.6 Let $g \in \mathbb{Q} R \widehat{G}$. Then

$$
g=\sum_{l=0}^{K-1} 2^{l-K} \cdot g_{l} \cdot(1-\chi) \cdot \prod_{\substack{0 \leq r \leq K-1 \\ r \neq l}}\left(1+\chi^{2^{r}}\right)
$$

for any elements $g_{l} \in \mathbb{Q} R \widehat{G}$ satisfying $\operatorname{pr}_{l}\left(g_{l}\right)=\operatorname{pr}_{l}(g)$.

If $\operatorname{pr}_{l}(g) \in 2^{2+K-l} \cdot \mathbb{Z}[\chi] /\left\langle 1+\chi^{2^{l}}\right\rangle$ we can choose $g_{l} \in 2^{2+K-l} \cdot R \widehat{G}$ satisfying $\operatorname{pr}_{l}\left(g_{l}\right)=\operatorname{pr}_{l}(g)$ and the lemma above shows $g \in 4 \cdot R \widehat{G}$. Motivated by this observation we want to analyze whether $\operatorname{pr}_{l}(g)$ lies in $2^{m} \cdot \mathbb{Z}[\chi] /\left\langle 1+\chi^{2^{l}}\right\rangle$ for some integer $m$. For this purpose we will introduce $w_{l}$-functions which are generalizations of the $p$-adic valuation for $p=2$.

Before we do so, we give the proof of Lemma 5.6 and consider an application (Lemma 5.7).

Proof of Lemma 5.6 We have $\mathbb{Q} R \widehat{G} \cong \mathbb{Q}[\chi] / I\langle K\rangle$ where $I\langle K\rangle$ was defined as $I\langle K\rangle:=\left\langle 1+\chi+\cdots+\chi^{2^{K}-1}\right\rangle$. Notice that $1+\chi+\cdots+\chi^{2^{K}-1}=\prod_{m=0}^{K-1}\left(1+\chi^{2^{m}}\right)$. Since the factors $1+\chi^{2^{m}}$ are mutually coprime in the principal ideal domain $\mathbb{Q}[\chi]$, it suffices to check the desired equality under the epimorphism $\operatorname{pr}_{m}$ for $0 \leq m \leq K-1$. In $\mathbb{Q}[\chi] /\left\langle 1+\chi^{2^{m}}\right\rangle$ we obtain

$$
\begin{aligned}
\operatorname{pr}_{m} & \left(\sum_{l=0}^{K-1} 2^{l-K} \cdot g_{l} \cdot(1-\chi) \cdot \prod_{\substack{0 \leq r \leq K-1 \\
r \neq l}}\left(1+\chi^{2^{r}}\right)\right) \\
& =\sum_{l=0}^{K-1} 2^{l-K} \cdot \operatorname{pr}_{m}(g) \cdot(1-\chi) \cdot \prod_{\substack{0 \leq r \leq K-1 \\
r \neq l}}\left(1+\chi^{2^{r}}\right) \\
& =2^{m-K} \cdot \operatorname{pr}_{m}(g) \cdot(1-\chi) \cdot \prod_{\substack{0 \leq r \leq K-1 \\
r \neq m}}\left(1+\chi^{2^{r}}\right) \\
& =2^{m-K} \cdot \operatorname{pr}_{m}(g) \cdot(1-\chi) \cdot\left(1+\chi+\cdots+\chi^{2^{m}-1}\right) \cdot \prod_{r=m+1}^{K-1}\left(1+(-1)^{2^{r-m}}\right) \\
& =2^{m-K} \cdot \operatorname{pr}_{m}(g) \cdot\left(1-\chi^{2^{m}}\right) \cdot 2^{K-1-m}=\operatorname{pr}_{m}(g) .
\end{aligned}
$$


As a warm-up in learning how to work with Lemma 5.6 we prove the following lemma needed in the proof of Corollary 4.14.

Lemma 5.7 Let $z \in \mathbb{Q} R \widehat{G}^{-}$. If $f \cdot z=0$ in $\mathbb{Q} R \widehat{G}^{+}$then $z=0$.

Proof It follows from Lemma 5.6 that it is sufficient to show $\operatorname{pr}_{l}(z)=0$ for all $0 \leq l \leq K-1$. We have $\operatorname{pr}_{l}(f) \cdot \operatorname{pr}_{l}(z)=\operatorname{pr}_{l}(f \cdot z)=0$. Notice that $\operatorname{pr}_{l}(f)$ is invertible for $l \geq 1$ since

$$
(1+\chi)^{-1}=\frac{1}{2} \cdot\left(1-\chi+\chi^{2}-\chi^{3}+\cdots-\chi^{2^{l}-1}\right) \in \mathbb{Q}[\chi] /\left\langle 1+\chi^{2^{l}}\right\rangle .
$$

This implies $\operatorname{pr}_{l}(z)=0$ for $l \geq 1$. Further recall that we can write $z \in \mathbb{Q} R \widehat{G}^{-}$as

$$
z=\sum_{r=1}^{N / 2-1} a_{r} \cdot\left(\chi^{r}-\chi^{N-r}\right)
$$

with $a_{r} \in \mathbb{Q}$. Since $\chi^{r}-\chi^{N-r}$ is a multiple of $1+\chi$, we conclude $\operatorname{pr}_{0}(z)=0$.

Now we proceed with the definition of the $w_{l}$-functions.

Definition 5.8 Let $\zeta_{2^{l+1}} s \in \mathbb{C}$ be a primitive $2^{l+1}$-th root of unity. Consider the ring of algebraic integers $\mathbb{Z}\left[\zeta_{2^{l+1} s}\right]$ in the cyclotomic field $\mathbb{Q}\left(\zeta_{2^{l+1}} s\right)$. The ideal $\mathcal{P}:=\left(1-\zeta_{2^{l+1} s}\right)$ in $\mathbb{Z}\left[\zeta_{2^{l+1} s}\right]$ is a prime ideal satisfying $\mathcal{P}^{2^{l}}=(2)$. Let $v_{\mathcal{P}}$ be the (exponential) valuation with respect to this prime ideal $\mathcal{P}$. Then the $w_{l}$-function of an element $g \in \mathbb{Q} R \widehat{G}$ is given by

$$
w_{l}(g)=\frac{1}{2^{l}} \cdot v_{\mathcal{P}}\left(\alpha\left(\operatorname{pr}_{l}(g)\right)\right) \in \frac{1}{2^{l}} \mathbb{Z} \cup\{\infty\}
$$

where $\alpha: \mathbb{Q}[\chi] /\left\langle 1+\chi^{2^{l}}\right\rangle \rightarrow \mathbb{Q}\left(\zeta_{2^{l+1} s}\right)$ is the isomorphism induced by $\chi \mapsto \zeta_{2^{l+1}} s$.

Roughly speaking, $w_{l}$ counts how many factors of 2 are contained in $\operatorname{pr}_{l}(g)$. The properties of the valuation $v_{\mathcal{P}}$ imply the following calculation rules for the $w_{l}$-function.

Lemma 5.9 Let $g_{1}, g_{2} \in \mathbb{Q} R \widehat{G}$ and $l \geq 0$.

(1) $w_{l}\left(g_{1} \cdot g_{2}\right)=w_{l}\left(g_{1}\right)+w_{l}\left(g_{2}\right)$.

(2) If $w_{l}\left(g_{1}\right) \neq w_{l}\left(g_{2}\right)$ then $w_{l}\left(g_{1}+g_{2}\right)=\min \left\{w_{l}\left(g_{1}\right), w_{l}\left(g_{2}\right)\right\}$.

(3) If $w_{l}\left(g_{1}\right)=w_{l}\left(g_{2}\right)$ then $w_{l}\left(g_{1}+g_{2}\right)>w_{l}\left(g_{1}\right)=w_{l}\left(g_{2}\right)$. 
Example 5.10 The reader is invited to calculate the $w_{l}$ for the following examples.

(1) If $q \in \mathbb{Q} \subset \mathbb{Q} R \widehat{G}$ then $w_{l}(q)$ coincides with the $p$-adic valuation for $p=2$. In particular, $w_{l}\left(2^{a}\right)=a$.

(2) $w_{l}(f)= \begin{cases}\infty & \text { when } l=0, \\ 0 & \text { when } l \geq 1\end{cases}$

(3) $w_{l}(f \pm 1)=1-2^{-l}$

(4) $w_{l}\left(f^{2}-1\right)=2-2^{1-l}$

(5) $w_{l}\left(f^{2}+1\right)= \begin{cases}0 & \text { when } l=0, \\ \infty & \text { when } l=1, \\ 1 & \text { when } l \geq 2\end{cases}$

(6) $w_{l}\left(f_{k}^{\prime}\right)=0$

Hints. (2): For $l=0$ use $f \equiv 0 \bmod 1+\chi$. For $l \geq 1$ use $f \cdot(1-\chi)=1+\chi$ and $1+\chi=2-(1-\chi)$. (3): Use $(1-\chi)(f+1)=2$ and $(1-\chi)(f-1)=2 \chi$. (4): Use (3). (5): For $l=1$ use $f^{2}+1 \equiv 0 \bmod 1+\chi^{2}$. For $l \neq 1$ use $f^{2}+1=\left(f^{2}-1\right)+2$ and (4). (6): Use $f_{k}^{\prime}, f_{k}^{\prime-1} \in R_{\widehat{G}}$ and the fact $w_{l}(g) \geq 0$ when $g \in R_{\widehat{G}}$.

We now come back to the initial question of this subsection: For a given $g \in \mathbb{Q} R \widehat{G}$ we want to decide whether $g$ lies in $4 \cdot R_{\widehat{G}}$ or not. The following theorem can answer this question in many cases.

Theorem 5.11 Let $g \in \mathbb{Q} R \widehat{G}$. Suppose that $\operatorname{pr}_{l}(g) \in 4 \cdot \mathbb{Z}[\chi] /\left\langle 1+\chi^{2^{l}}\right\rangle$ for all $0 \leq l \leq K-1$.

(1) If $w_{l}(g) \geq 2+K-l-2^{-l}$ for all $0 \leq l \leq K-1$ then $g \in 4 \cdot R \widehat{G}$.

(2) If there exist $h \in R \widehat{G}$ and $0 \leq l^{\prime} \leq K-1$ such that

$$
\begin{aligned}
& \quad w_{l}(g)+w_{l}(h) \geq 2+K-l-2^{-l} \text { for all } l \in\{0,1, \ldots, K-1\}-\left\{l^{\prime}\right\}, \\
& w_{l^{\prime}}(g)+w_{l^{\prime}}(h)<2+K-l^{\prime}-2^{-l^{\prime}}, \\
& \text { then } g \notin 4 \cdot R_{\widehat{G}} .
\end{aligned}
$$

Proof (1) The assumption $w_{l}(g) \geq 2+K-l-2^{-l}$ implies $w_{l}((1-\chi) \cdot g) \geq 2+K-l$. Hence, for all $0 \leq l \leq K-1$ there exist $z_{l} \in \mathbb{Z}[\chi]$ such that

$$
\operatorname{pr}_{l}((1-\chi) \cdot g)=2^{2+K-l} \cdot z_{l}(\chi) \in \mathbb{Q}[\chi] /\left\langle 1+\chi^{2^{l}}\right\rangle .
$$


Using Lemma 5.6 we conclude

$$
(1-\chi) \cdot g=\sum_{l=0}^{K-1} 2^{l-K} \cdot\left(2^{2+K-l} \cdot z_{l}(\chi)\right) \cdot(1-\chi) \cdot \prod_{\substack{0 \leq r \leq K-1 \\ r \neq l}}\left(1+\chi^{2^{r}}\right) \quad \text { in } \mathbb{Q} R \widehat{G}
$$

and hence $g=4 \cdot \sum_{l=0}^{K-1} \cdot z_{l}(\chi) \cdot \prod_{\substack{0 \leq r \leq K-1 \\ r \neq l}}\left(1+\chi^{2^{r}}\right) \in 4 \cdot R \widehat{G}$.

(2) We give a proof by contradiction. Assume that $g \in 4 \cdot R \widehat{G}$ and define

$$
a:=\min \left\{m \in \mathbb{Z} \mid m+w_{l^{\prime}}(g)+w_{l^{\prime}}(h) \geq 2+K-l^{\prime}-2^{-l^{\prime}}\right\} .
$$

Notice that $a \geq 1$. We have

$$
\begin{aligned}
& w_{l}\left((1-\chi) \cdot 2^{a} \cdot g \cdot h\right) \geq 3+K-l \quad \text { for all } l \in\{0,1, \ldots, K-1\}-\left\{l^{\prime}\right\} \\
& w_{l^{\prime}}\left((1-\chi) \cdot 2^{a} \cdot g \cdot h\right) \geq 2+K-l^{\prime} .
\end{aligned}
$$

We conclude that there exist $z_{l} \in 2 \cdot \mathbb{Z}[\chi]$ for all $l \in\{0,1, \ldots, K-1\}-\left\{l^{\prime}\right\}$ and $z_{l^{\prime}} \in \mathbb{Z}[\chi]$ satisfying

$$
\operatorname{pr}_{l}\left((1-\chi) \cdot 2^{a} \cdot g \cdot h\right)=2^{2+K-l} \cdot z_{l}(\chi) \in \mathbb{Q}[\chi] /\left\langle 1+\chi^{2^{l}}\right\rangle .
$$

Lemma 5.6 implies

$$
(1-\chi) \cdot 2^{a} \cdot g \cdot h=\sum_{l=0}^{K-1} 2^{l-K} \cdot 2^{2+K-l} \cdot z_{l}(\chi) \cdot(1-\chi) \cdot \prod_{\substack{0 \leq r \leq K-1 \\ r \neq l}}\left(1+\chi^{2^{r}}\right)
$$

and hence $\quad 2^{a} \cdot g \cdot h=\sum_{l=0}^{K-1} 4 \cdot z_{l}(\chi) \cdot \prod_{\substack{0 \leq r \leq K-1 \\ r \neq l}}\left(1+\chi^{2^{r}}\right) \quad$ in $\mathbb{Q} R \widehat{G}$.

Since $g \cdot h \in 4 \cdot R \widehat{G}$ there exists $y \in \mathbb{Z}[\chi]$ such that $g \cdot h$ and $4 \cdot y$ coincide in $\mathbb{Q}[\chi] / I\langle K\rangle$. We get

$$
2^{a} \cdot y(\chi)=\sum_{l=0}^{K-1} z_{l}(\chi) \cdot \prod_{\substack{0 \leq r \leq K-1 \\ r \neq l}}\left(1+\chi^{2^{r}}\right) \quad \text { in } \mathbb{Q}[\chi] / I\langle K\rangle .
$$

Hence there exists $q \in \mathbb{Q}[\chi]$ with

$$
2^{a} \cdot y(\chi)=\sum_{l=0}^{K-1} z_{l}(\chi) \cdot \prod_{\substack{0 \leq r \leq K-1 \\ r \neq l}}\left(1+\chi^{2^{r}}\right)+q(\chi) \cdot\left(1+\chi+\cdots+\chi^{N-1}\right) .
$$


The equation above implies $q(\chi) \cdot\left(1+\chi+\cdots+\chi^{N-1}\right) \in \mathbb{Z}[\chi]$ and hence $q \in \mathbb{Z}[\chi]$. Under the epimorphism $\mathbb{Z}[\chi] \rightarrow \mathbb{Z}_{2}[\chi]$ this equation becomes

$$
\begin{aligned}
0 & =\sum_{l=0}^{K-1} \overline{z_{l}}(\chi) \cdot \prod_{\substack{0 \leq r \leq K-1 \\
r \neq l}}\left(1+\chi^{2^{r}}\right)+\bar{q}(\chi) \cdot\left(1+\chi+\cdots+\chi^{N-1}\right) \\
& =\overline{z_{l^{\prime}}}(\chi) \cdot \prod_{\substack{0 \leq r \leq K-1 \\
r \neq l^{\prime}}}\left(1+\chi^{2^{r}}\right)+\bar{q}(\chi) \cdot \prod_{r=0}^{K-1}\left(1+\chi^{2^{r}}\right) .
\end{aligned}
$$

Hence $\overline{z_{l^{\prime}}}(\chi)=-\bar{q}(\chi) \cdot\left(1+\chi^{2^{l^{\prime}}}\right)=-\bar{q}(\chi) \cdot(1+\chi)^{2^{l^{\prime}}}$ in $\mathbb{Z}_{2}[\chi]$. This implies $w_{l^{\prime}}\left(z_{l^{\prime}}\right) \geq 1$. We finally get

$$
\begin{aligned}
(a-1)+w_{l^{\prime}}(g)+w_{l^{\prime}}(h) & =w_{l^{\prime}}\left((1-\chi) \cdot 2^{a} \cdot g \cdot h\right)-1-2^{-l^{\prime}} \\
& =w_{l^{\prime}}\left(2^{2+K-l^{\prime}} \cdot z_{l}^{\prime}(\chi)\right)-1-2^{-l^{\prime}} \\
& =w_{l^{\prime}}\left(z_{l}^{\prime}(\chi)\right)+1+K-l^{\prime}-2^{-l^{\prime}} \\
& \geq 2+K-l^{\prime}-2^{-l^{\prime}}
\end{aligned}
$$

which contradicts the minimality of $a$.

\section{$5.2 c_{K}$-technology}

In this subsection we introduce the $c_{K}$-functions which measure the "failure" of a polynomial $g \in \mathbb{Q} R \widehat{G}$ to be in $4 \cdot R \widehat{G}$ and we show their two interesting properties.

Definition 5.12 Let $G=\mathbb{Z}_{2 K}$. Define the function $c_{K}: \mathbb{Q} R \widehat{G} \longrightarrow \mathbb{N}_{0} \cup\{\infty\}$ by

$$
c_{K}(g):=\min \left\{c \in \mathbb{N}_{0} \mid g(\chi) \cdot(1-\chi)^{c} \in 4 \cdot \mathbb{Z}[\chi] / I\langle K\rangle\right\} .
$$

If the set on the right hand side is empty then we set $c_{K}(g):=\infty$.

Lemma 5.13 Let $g \in 2 \cdot R \overline{\widehat{G}}$ where $G=\mathbb{Z}_{2^{K}}$. Then

$$
c_{K}(g) \in\{0\} \cup\left\{1,3,5, \ldots, 2^{K}-1\right\} .
$$

Proof The element $g \in 2 \cdot R_{\widehat{G}}$ can be written as

$$
g(\chi)=2 \cdot \sum_{k=1}^{2^{K-1}} a_{k} \cdot\left(\chi^{k}-\chi^{-k}\right)
$$


with $a_{k} \in \mathbb{Z}$. We set

$$
\bar{g}(\chi):=\sum_{k=1}^{2^{K-1}} a_{k} \cdot\left(\chi^{k}-\chi^{-k}\right) \in \mathbb{Z}_{2}[\chi] / I\langle K\rangle .
$$

Now suppose that $c_{K}(g) \neq 0$ ie $\bar{g} \neq 0$. Since

$$
\chi^{k}-\chi^{-k}=\left(\chi-\chi^{-1}\right) \cdot\left(\chi^{1-k}+\chi^{3-k}+\cdots+\chi^{k-3}+\chi^{k-1}\right),
$$

any element $y \in \mathbb{Z}_{2}[\chi] / I\langle K\rangle$ of the shape $y(\chi)=\sum_{k=1}^{m} c_{k} \cdot\left(\chi^{k}-\chi^{-k}\right)$ can be written as

$$
y(\chi)=\left(\chi-\chi^{-1}\right) \cdot\left(c_{0}^{\prime}+\sum_{k=1}^{m-1} c_{k}^{\prime} \cdot\left(\chi^{k}-\chi^{-k}\right)\right) .
$$

Now, we transform $\bar{g}$ in this way and repeat the transformation as long as the occurring $c_{0}^{\prime}$ is zero. We finally get

$$
\bar{g}(\chi)=\left(\chi-\chi^{-1}\right)^{n} \cdot\left(1+\sum_{k=1}^{2^{K-1}-n} b_{k} \cdot\left(\chi^{k}-\chi^{-k}\right)\right) .
$$

We want to prove $c_{K}(g)=c:=2^{K}-2 n-1$. Notice that we have in $\mathbb{Z}_{2}[\chi]$

$$
\begin{aligned}
1+\chi+\cdots+\chi^{2^{K}-1} & =\prod_{r=1}^{K-1}\left(1+\chi^{2^{r}}\right)=\prod_{r=1}^{K-1}(1-\chi)^{2^{r}}=(1-\chi)^{2^{K}-1} \\
\left(\chi-\chi^{-1}\right)^{n} & =\left(\chi^{-1} \cdot(1-\chi)^{2}\right)^{n}=\chi^{-n} \cdot(1-\chi)^{2 n} .
\end{aligned}
$$

Therefore, we calculate in $\mathbb{Z}_{2}[\chi] / I\langle K\rangle$

$$
\left(\chi-\chi^{-1}\right)^{n} \cdot(1-\chi)^{c}=\chi^{-n} \cdot(1-\chi)^{2^{K}-1}=0 .
$$

This implies $\bar{g}(\chi) \cdot(1-\chi)^{c}=0$ in $\mathbb{Z}_{2}[\chi] / I\langle K\rangle$ and hence

$$
g(\chi) \cdot(1-\chi)^{c} \in 4 \cdot \mathbb{Z}[\chi] / I\langle K\rangle .
$$

It remains to show

$$
g(\chi) \cdot(1-\chi)^{c-1} \notin 4 \cdot \mathbb{Z}[\chi] / I\langle K\rangle .
$$

We prove this by contradiction. Suppose $g(\chi) \cdot(1-\chi)^{c-1} \in 4 \cdot \mathbb{Z}[\chi] / I\langle K\rangle$ which implies $\bar{g}(\chi) \cdot(1-\chi)^{c-1}=0$ in $\mathbb{Z}_{2}[\chi] / I\langle K\rangle$. This means that there exists $q \in \mathbb{Z}_{2}[\chi]$ with

$\left(\chi-\chi^{-1}\right)^{n} \cdot\left(1+\sum_{k=1}^{2^{K-1}-n} b_{k} \cdot\left(\chi^{k}-\chi^{-k}\right)\right) \cdot(1-\chi)^{c-1}=q(\chi) \cdot\left(1+\chi+\cdots+\chi^{2^{K}-1}\right)$ 
We conclude in $\mathbb{Z}_{2}[\chi]$

$$
\chi^{-n} \cdot(1-\chi)^{2 n} \cdot\left(1+\sum_{k=1}^{2^{K-1}-n} b_{k} \cdot\left(\chi^{k}-\chi^{-k}\right)\right) \cdot(1-\chi)^{c-1}=q(\chi) \cdot(1-\chi)^{2^{K}-1}
$$

and hence

$$
\chi^{-n} \cdot\left(1+\sum_{k=1}^{2^{K-1}-n} b_{k} \cdot\left(\chi^{k}-\chi^{-k}\right)\right)=q(\chi) \cdot(1-\chi) .
$$

This implies the desired contradiction

$$
1=1^{-n} \cdot\left(1+\sum_{k=1}^{2^{K-1}-n} b_{k} \cdot\left(1^{k}-1^{-k}\right)\right)=q(1) \cdot(1-1)=0 \quad \text { in } \mathbb{Z}_{2} .
$$

Lemma 5.14 Let $g_{i} \in 2 \cdot \mathbb{Z}[\chi] / I\langle K\rangle$ for $i=1,2$ such that $c_{K}\left(g_{1}\right)=c_{K}\left(g_{2}\right)>0$. Then we have

$$
c_{K}\left(g_{1}+g_{2}\right)<c_{K}\left(g_{1}\right)=c_{K}\left(g_{2}\right) \text {. }
$$

Proof We set $c:=c_{K}\left(g_{1}\right)=c_{K}\left(g_{2}\right)$. Since $2 \cdot g_{i}(\chi) \cdot(1-\chi)^{c-1} \in 4 \cdot \mathbb{Z}[\chi] / I\langle K\rangle$, there exist polynomials $h_{i} \in \mathbb{Z}[\chi](i=1,2)$ such that $2 \cdot g_{i}(\chi) \cdot(1-\chi)^{c-1}$ and $4 \cdot h_{i}(\chi)$ coincide modulo $I\langle K\rangle$. We can require that $\operatorname{deg}\left(h_{i}\right) \leq 2^{K}-2$. Let $\overline{h_{i}}$ be the image of $h_{i}$ under the epimorphism $\mathbb{Z}[\chi] \rightarrow \mathbb{Z}_{2}[\chi]$. Notice that $1+\chi+\cdots+\chi^{2^{K}-1}$ divides $\overline{h_{i}}(\chi) \cdot(1-\chi)$ in $\mathbb{Z}_{2}[\chi]$ because of $g_{i}(\chi) \cdot(1-\chi)^{c} \in 4 \cdot \mathbb{Z}[\chi] / I\langle K\rangle$. In $\mathbb{Z}_{2}[\chi]$ we have

$$
1+\chi+\cdots+\chi^{2^{K}-1}=\prod_{r=0}^{K-1}\left(1+\chi^{2^{r}}\right)=\prod_{r=0}^{K-1}(1+\chi)^{2^{r}}=(1+\chi)^{2^{K}-1} .
$$

Therefore, $(1+\chi)^{2^{K}-2}$ divides $\overline{h_{i}}(\chi)$. Since $g_{i}(\chi) \cdot(1-\chi)^{c-1} \notin 4 \cdot \mathbb{Z}[\chi] / I\langle K\rangle$, we have $\overline{h_{i}}(\chi) \neq 0$. We conclude from $\operatorname{deg}\left(\overline{h_{i}}\right) \leq \operatorname{deg}\left(h_{i}\right) \leq 2^{K}-2$ that $\overline{h_{i}}(\chi)=(1+\chi)^{2^{K}-2}$. Therefore,

$$
\overline{h_{1}}(\chi)+\overline{h_{2}}(\chi)=2 \cdot(1+\chi)^{2^{K}-2}=0 \quad \text { in } \mathbb{Z}_{2}[\chi] .
$$

This implies $h_{1}(\chi)+h_{2}(\chi) \in 2 \cdot \mathbb{Z}[\chi]$. In $\mathbb{Q}[\chi] / I\langle K\rangle$ we finally conclude

$$
\left(g_{1}(\chi)+g_{2}(\chi)\right) \cdot(1-\chi)^{c-1}=2 \cdot\left(h_{1}(\chi)+h_{2}(\chi)\right) \in 4 \cdot \mathbb{Z}[\chi] / I\langle K\rangle .
$$

Lemma 5.15 Let $g_{i} \in 2 \cdot \mathbb{Z}[\chi] / I\langle K\rangle$ for $i=1$, 2 such that $c_{K}\left(g_{1}\right)>c_{K}\left(g_{2}\right)$. Then

$$
c_{K}\left(g_{1}+g_{2}\right)=c_{K}\left(g_{1}\right)>c_{K}\left(g_{2}\right) \text {. }
$$

Proof Obviously, we have

$$
c_{K}\left(g_{1}+g_{2}\right) \leq \max \left\{c_{K}\left(g_{1}\right), c_{K}\left(g_{2}\right)\right\}=c_{K}\left(g_{1}\right) .
$$


The inequality $c_{K}\left(g_{1}+g_{2}\right) \geq c_{K}\left(g_{1}\right)$ can be concluded from

$$
c_{K}\left(g_{1}\right)=c_{K}\left(\left(g_{1}+g_{2}\right)-g_{2}\right) \leq \max \left\{c_{K}\left(g_{1}+g_{2}\right), c_{K}\left(g_{2}\right)\right\} .
$$

\subsection{Inductive properties}

Lemma 5.16 Let $g \in \mathbb{Q}[\chi] / I\langle K+1\rangle$ such that $\operatorname{pr}_{K}(g) \in 4 \cdot \mathbb{Z}[\chi] /\left\langle 1+\chi^{2^{K}}\right\rangle$. Then

$$
g \in 4 \cdot \mathbb{Z}[\chi] / I\langle K\rangle \Longleftrightarrow 2 g \in 4 \cdot \mathbb{Z}[\chi] / I\langle K+1\rangle .
$$

Proof Assume first $g \in 4 \cdot \mathbb{Z}[\chi] / I\langle K\rangle$. Let $h \in \mathbb{Z}[\chi]$ be such that $4 h$ and $g$ coincide in $\mathbb{Q}[\chi] /\left\langle 1+\chi^{2^{K}}\right\rangle$ and let $k \in \mathbb{Z}[\chi]$ such that $4 k$ and $g$ coincide in $\mathbb{Q}[\chi] / I\langle K\rangle$. Then we obtain in $\mathbb{Q}[\chi] / I\langle K+1\rangle$ the equation

$$
2 \cdot g(\chi)=4 \cdot\left(1+\chi^{2^{K}}\right) \cdot k(\chi)+4 \cdot\left(1-\chi^{2^{K}}\right) \cdot h(\chi) .
$$

which shows $2 g \in 4 \cdot \mathbb{Z}[\chi] / I\langle K+1\rangle$.

Now assume $2 g \in 4 \cdot \mathbb{Z}[\chi] / I\langle K+1\rangle$. We want to show $g \in 4 \cdot \mathbb{Z}[\chi] / I\langle K\rangle$. Let $h \in \mathbb{Z}[\chi]$ be again such that $4 h$ and $g$ coincide in $\mathbb{Q}[\chi] /\left\langle 1+\chi^{2^{K}}\right\rangle$ and let $k \in \mathbb{Z}[\chi]$ be such that $4 k$ and $2 g$ (resp. $2 k$ and $g$ ) coincide in $\mathbb{Q}[\chi] / I\langle K+1\rangle$. Then $2 \cdot k(\chi)$ and $k(\chi) \cdot\left(1+\chi^{2^{K}}\right)+2 \cdot h(\chi) \cdot\left(1-\chi^{2^{K}}\right)$ coincide in $\mathbb{Q}[\chi] / I\langle K\rangle$ and in $\mathbb{Q}[\chi] /\left\langle 1+\chi^{2^{K}}\right\rangle$ and hence also in $\mathbb{Q}[\chi] / I\langle K+1\rangle$. Therefore there exists an $r \in \mathbb{Q}[\chi]$ with

$$
2 \cdot k(\chi)=k(\chi) \cdot\left(1+\chi^{2^{K}}\right)+2 \cdot h(\chi) \cdot\left(1-\chi^{2^{K}}\right)+r(\chi) \cdot\left(1+\chi+\cdots+\chi^{2^{K+1}-1}\right) .
$$

We conclude $r \in \mathbb{Z}[\chi]$. Under the epimorphism $\mathbb{Z}[\chi] \rightarrow \mathbb{Z}_{2}[\chi]$ we get

$$
0=\bar{k}(\chi) \cdot\left(1+\chi^{2^{K}}\right)+\bar{r}(\chi) \cdot\left(1+\chi+\cdots+\chi^{2^{K+1}-1}\right)
$$

and hence

$$
0=\bar{k}(\chi)+\bar{r}(\chi) \cdot\left(1+\chi+\cdots+\chi^{2^{K}-1}\right) .
$$

We set $s(\chi):=k(\chi)+r(\chi) \cdot\left(1+\chi+\cdots+\chi^{2^{K}-1}\right) \in \mathbb{Z}[\chi]$. The vanishing of $s$ under the epimorphism $\mathbb{Z}[\chi] \rightarrow \mathbb{Z}_{2}[\chi]$ implies the existence of $t \in \mathbb{Z}[\chi]$ with $2 t=s$. We conclude in $\mathbb{Q}[\chi] / I\langle K\rangle$

$$
g=2 k=2 s=4 t .
$$

This shows $g \in 4 \cdot \mathbb{Z}[\chi] / I\langle K\rangle$.

Corollary 5.17 Let $g \in \mathbb{Q}[\chi] / I\langle K+1\rangle$ such that $\operatorname{pr}_{K}(g) \in 4 \cdot \mathbb{Z}[\chi] /\left\langle 1+\chi^{2^{K}}\right\rangle$. Then we have

$$
c_{K+1}(2 g)=c_{K}(g) \text {. }
$$




\subsection{Good polynomials}

In this subsection we define polynomials $r_{n}^{-}$which are the "best" polynomials in the sense they are polynomials with leading coefficient 1 yielding elements in $4 \cdot \mathbb{Z}[\chi] / I\langle K\rangle$ for a large $K$ in comparison with the other polynomials of the same degree with leading coefficient 1 . The path to the definition might be seen as a 'brute force' approach, on the other hand it is a systematic approach as we will try to convey shortly. In order to understand the overall strategy of the proof of Theorem 5.4 it might be better to skip this subsection in the first reading, except Proposition 5.24, which describes the properties of the polynomials $r_{n}^{-}$and which is all that is needed for the proof of Theorem 5.4 in the next subsection.

The method in this subsection is to combine the $w_{l}$-technology, the $c_{K}$-technology and the induction technology. In the first part we define auxiliary polynomials $p_{k}$ (Definition 5.18) which are used to define polynomials denoted $q_{n}$ (Definition 5.21). These polynomials are defined so that they have obviously large $w_{l}$-functions (see Corollary 5.20 and Lemma 5.23). In view of the $c_{K}$-technology and the induction procedure it is important to understand all those properties of the polynomials $q_{n}$ which are listed in Proposition 5.22. The next idea is to use the $c_{K}$-technology, the induction procedure and the known properties of the polynomials $q_{n}$ to see whether there are better polynomials than $q_{n}$. Such a systematic analysis is done in Proposition 5.24. Using it we obtain the best polynomials $r_{n}^{-}$(Definition 5.26) and their properties (Corollary 5.27).

Polynomials $p_{k}$ Notice that the for any $q(x) \in \mathbb{Q}[x]$ we get

$$
w_{0}\left(8 \cdot f_{k}^{\prime} \cdot f \cdot q\left(f^{2}\right)\right)=\infty
$$

since $w_{0}(f)=\infty$ because of $(1+\chi) \mid f$. Further notice that for $p_{1}(x):=x+1$ we have

$$
p_{1}\left(f^{2}\right)=f^{2}+1=2 \cdot \frac{1+\chi^{2}}{(1-\chi)^{2}} .
$$

Hence $\left(1+\chi^{2}\right) \mid p_{1}\left(f^{2}\right)$ in $\mathbb{Q}[\chi] / I\langle K\rangle$ and $w_{1}\left(8 \cdot f_{k}^{\prime} \cdot f \cdot p_{1}\left(f^{2}\right)\right)=\infty$. Further observe that

$$
\frac{\left(f^{2}+1\right)^{2}}{4 \cdot f^{2}}=\frac{\left(1+\chi^{2}\right)^{2}}{\left(1-\chi^{2}\right)^{2}} .
$$

Motivated by that we make the following: 
Definition 5.18 Let $p_{1}(x):=x+1 \in \mathbb{Z}[x]$. For $k \in \mathbb{N}$ define inductively

$$
p_{k+1}(x):=p_{k}\left(\frac{(x+1)^{2}}{4 x}\right) \cdot(4 x)^{2^{k-1}} \in \mathbb{Z}[x] .
$$

Notice that $p_{k}(x)$ is a polynomial in $\mathbb{Z}[x]$ of degree $2^{k-1}$.

Theorem 5.19 We have

$$
p_{k}\left(f^{2}\right)=2^{2^{k}-1} \cdot \frac{1+\chi^{2^{k}}}{(1-\chi)^{2^{k}}} \in \mathbb{Q}[\chi] / I\langle K\rangle \quad \text { for } k \in \mathbb{N} .
$$

Proof It suffices to prove the equality

$$
p_{k}\left(f^{2}\right)=2^{2^{k}-1} \cdot \frac{1+\chi^{2^{k}}}{(1-\chi)^{2^{k}}}
$$

in the field of rational functions $\mathbb{Q}(\chi)$. The proof now goes by induction with respect to $k \in \mathbb{N}$. The case $k=1$ is proved by the identity (5-5). Now the induction step. Let $\alpha: \mathbb{Q}(\chi) \longrightarrow \mathbb{Q}(\chi)$ be the homomorphism given by $\chi \mapsto \chi^{2}$. We calculate:

$$
\begin{aligned}
& p_{k+1}\left(f^{2}\right)=p_{k}\left(\frac{\left(f^{2}+1\right)^{2}}{4 \cdot f^{2}}\right) \cdot\left(4 f^{2}\right)^{2^{k-1}} \\
& =p_{k}\left(\left(\frac{1+\chi^{2}}{1-\chi^{2}}\right)^{2}\right) \cdot 2^{2^{k}} \cdot f^{2^{k}} \\
& =p_{k}\left((\alpha(f))^{2}\right) \cdot 2^{2^{k}} \cdot f^{2^{k}} \\
& =\alpha\left(p_{k}\left(f^{2}\right)\right) \cdot 2^{2^{k}} \cdot f^{2^{k}} \\
& =\frac{1+\left(\chi^{2}\right)^{2^{k}}}{\left(1-\chi^{2}\right)^{2^{k}}} \cdot 2^{2^{k}-1} \cdot 2^{2^{k}} \cdot \frac{(1+\chi)^{2^{k}}}{(1-\chi)^{2^{k}}}
\end{aligned}
$$

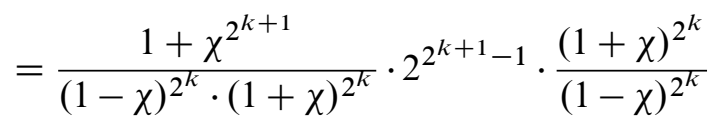

$$
\begin{aligned}
& =2^{2^{k+1}-1} \cdot \frac{1+\chi^{2^{k+1}}}{(1-\chi)^{2^{k+1}}}
\end{aligned}
$$

Corollary 5.20 We have

(1) $w_{l}\left(p_{k}\left(f^{2}\right)\right)=\infty$ when $l=k$,

(2) $w_{l}\left(p_{k}\left(f^{2}\right)\right)=2^{k}-1$ when $l>k$. 
Proof The first item is immediate from the formula of the previous theorem. For the second item note that $1+\chi^{2^{k}} \equiv(1-\chi)^{2^{k}} \bmod 2$. It follows that $w_{l}\left(1+\chi^{2^{k}}\right)=$ $w_{l}\left((1-\chi)^{2^{k}}\right)$ for $l>k$ and hence

$$
w_{l}\left(\frac{1+\chi^{2^{k}}}{(1-\chi)^{2^{k}}}\right)=0 \quad \text { for } l>k .
$$

Finally use the formula of the previous theorem and the product formula for $w_{l}$.

Polynomials $q_{n}$ Now we are ready to introduce the polynomials $q_{n}$ which will be good in the already mentioned sense. The idea is that we get good polynomials when we multiply the polynomials $p_{k}$ from the previous definition.

Definition 5.21 Let $n \geq 0$. Define $a(n), b(n) \geq 0$ as the integers satisfying

$$
n+1=2^{a(n)}+b(n) \quad \text { with } \quad 0 \leq b(n) \leq 2^{a(n)}-1 .
$$

Define

$$
q_{n}(x):=\prod_{r=1}^{a(n)} p_{r}(x) \cdot(x-1)^{b(n)} .
$$

Proposition 5.22 Let $n \geq 0, k \geq 1$ and $m \in\{1,2\}$. We have

$$
\begin{aligned}
& 8 \cdot f_{k}^{\prime} \cdot f^{m} \cdot q_{n}\left(f^{2}\right) \in 4 \cdot \mathbb{Z}[\chi] / I\langle 2 n+1\rangle, \\
& 8 \cdot f_{k}^{\prime} \cdot f^{m} \cdot q_{n}\left(f^{2}\right) \in 4 \cdot \mathbb{Z}[\chi] / I\langle 2 n+2\rangle \Longleftrightarrow b(n)=0 .
\end{aligned}
$$

Moreover, we have

$$
\begin{aligned}
c_{2 n+2}\left(8 \cdot f_{k}^{\prime} \cdot f^{m} \cdot q_{n}\left(f^{2}\right)\right) & =\max \{2 b(n)-1,0\}, \\
c_{2 n+3}\left(8 \cdot f_{k}^{\prime} \cdot f^{m} \cdot q_{n}\left(f^{2}\right)\right) & =2 n+1, \\
c_{2 n+2+s}\left(8 \cdot f_{k}^{\prime} \cdot f^{m} \cdot q_{n}\left(f^{2}\right)\right) & \leq 2 n+1+2^{a(n)}\left(2^{s}-2\right) \text { for all } s \geq 1 .
\end{aligned}
$$

We use the $w_{l}$-technology for which we need:

Lemma 5.23 Let $n \geq 0, k \geq 1$ and $m \in\{1,2\}$. We have

$$
w_{l}\left(8 \cdot f_{k}^{\prime} \cdot f^{m} \cdot q_{n}\left(f^{2}\right)\right)= \begin{cases}\infty & l \leq a(n), \\ 2 n+3-a(n)-\frac{b(n)}{2^{l-1}} & l \geq a(n)+1\end{cases}
$$

Proof Use the formulas from Lemma 5.9, Example 5.10 and Corollary 5.20. 
Proof of Proposition 5.22 The desired results are obtained using the criteria from Theorem 5.11.

$$
\begin{aligned}
w_{l}\left(8 \cdot f_{k}^{\prime} \cdot f^{m} \cdot q_{n}\left(f^{2}\right)\right)-(2+2 n+1-l & \left.-2^{-l}\right) \\
& = \begin{cases}\infty & l \leq a(n), \\
l-a(n)-\frac{2 b(n)-1}{2^{l}} \geq 0 & l \geq a(n)+1\end{cases}
\end{aligned}
$$

implies

$$
8 \cdot f_{k}^{\prime} \cdot f^{m} \cdot q_{n}\left(f^{2}\right) \in 4 \cdot \mathbb{Z}[\chi] / I\langle 2 n+1\rangle .
$$

For $b(n)>0$ we have

$$
\begin{aligned}
w_{l}\left(8 \cdot f_{k}^{\prime} \cdot f^{m} \cdot q_{n}\left(f^{2}\right) \cdot(1-\chi)^{2 b(n)-2}\right)- & \left(2+2 n+2-l-2^{-l}\right) \\
& = \begin{cases}\infty & l \leq a(n), \\
-\frac{1}{2^{a(n)+1}}<0 & l=a(n)+1, \\
l-(a(n)+1)-\frac{1}{2^{l}} \geq 0 & l \geq a(n)+2,\end{cases}
\end{aligned}
$$

and $\quad w_{l}\left(8 \cdot f_{k}^{\prime} \cdot f^{m} \cdot q_{n}\left(f^{2}\right) \cdot(1-\chi)^{2 b(n)-1}\right)-\left(2+2 n+2-l-2^{-l}\right)$

$$
= \begin{cases}\infty & l \leq a(n), \\ l-(a(n)+1) \geq 0 & l \geq a(n)+1,\end{cases}
$$

which implies $\quad c_{2 n+2}\left(8 \cdot f_{k}^{\prime} \cdot f^{m} \cdot q_{n}\left(f^{2}\right)\right)=2 b(n)-1$.

For $b(n)=0$ we have

$$
\begin{aligned}
w_{l}\left(8 \cdot f_{k}^{\prime} \cdot f^{m} \cdot q_{n}\left(f^{2}\right)\right)-(2+2 n+2- & \left.l-2^{-l}\right) \\
& = \begin{cases}\infty & l \leq a(n), \\
l-(a(n)+1)+\frac{1}{2^{l}} \geq 0 & l \geq a(n)+1,\end{cases}
\end{aligned}
$$

which implies $\quad 8 \cdot f_{k}^{\prime} \cdot f^{m} \cdot q_{n}\left(f^{2}\right) \in 4 \cdot \mathbb{Z}[\chi] / I\langle 2 n+2\rangle$.

From $w_{l}\left(8 \cdot f_{k}^{\prime} \cdot f^{m} \cdot q_{n}\left(f^{2}\right) \cdot(1-\chi)^{2 n}\right)-\left(2+2 n+3-l-2^{-l}\right)$

$$
= \begin{cases}\infty & l \leq a(n), \\ -\frac{1}{2^{a(n)+1}}<0 & l=a(n)+1, \\ l-(a(n)+2)+\frac{2^{a(n)+1}-1}{2^{l}} \geq 0 & l \geq a(n)+2,\end{cases}
$$

we conclude

$$
c_{2 n+3}\left(8 \cdot f_{k}^{\prime} \cdot f^{m} \cdot q_{n}\left(f^{2}\right)\right) \geq 2 n+1 \text {. }
$$

It remains to show

$$
c_{2 n+2+s}\left(8 \cdot f_{k}^{\prime} \cdot f^{m} \cdot q_{n}\left(f^{2}\right)\right) \leq 2 n+1+2^{a(n)}\left(2^{s}-2\right) \text { for all } s \geq 1 .
$$




$$
\begin{aligned}
& w_{l}\left(8 \cdot f_{k}^{\prime} \cdot f^{m} \cdot q_{n}\left(f^{2}\right) \cdot(1-\chi)^{2 n+1+2^{a(n)}\left(2^{s}-2\right)}\right)-\left(2+2 n+2+s-l-2^{-l}\right) \\
& = \begin{cases}\infty & l \leq a(n), \\
l-a(n)-s-1+2^{a(n)+s-l} & l \geq a(n)+1 .\end{cases}
\end{aligned}
$$

We set $z:=a(n)+s-l$ and have to show $2^{z} \geq z+1$ for all $z \in \mathbb{Z}$. This is obviously true for $z \leq-1$. The statement for $z \geq 0$ follows by induction.

Polynomials $\tilde{q}_{n}$ Notice that the polynomials $q_{n}$ have slightly better properties when $b(n)=0$. This suggests that there might exist better polynomials than $q_{n}$ when $b(n)>0$. This turns out to be true, there exist polynomials $r_{n}^{-}$of degree $n$ with leading coefficient 1 such that

$$
8 \cdot f_{k}^{\prime} \cdot f^{m} \cdot r_{n}^{-}\left(f^{2}\right) \in 4 \cdot \mathbb{Z}[\chi] / I\langle 2 n+2\rangle .
$$

Their construction needs some preparation. They are obtained inductively. The crucial inductive step is based on the following proposition. The idea is motivated by the properties of $q_{n}$ when $b(n)=0$ and is based on the following observation: If we assume for a given $n \in \mathbb{N}$ with $b(n)>0$ the existence of polynomials $\widetilde{q}_{l}$ for $l \leq\left\lfloor\frac{n}{2}\right\rfloor-1$ which are slightly better than $q_{l}$ then we are able to conclude the existence of a $\tilde{q}_{n}$ which is also better than $q_{n}$.

Proposition 5.24 Let $n \geq 0, k \geq 1$ and $m \in\{1,2\}$. Let $\widetilde{q}_{l} \in \mathbb{Z}[\chi]$ be polynomials for $0 \leq l \leq\left\lfloor\frac{n}{2}\right\rfloor-1$ such that

$$
\begin{gathered}
8 \cdot f_{k}^{\prime} \cdot f^{m} \cdot \tilde{q}_{l}\left(f^{2}\right)=4 \cdot \mathbb{Z}[\chi] / I\langle 2 l+2\rangle, \\
c_{2 l+3}\left(8 \cdot f_{k}^{\prime} \cdot f^{m} \cdot \widetilde{q}_{l}\left(f^{2}\right)\right)=2 l+1, \\
c_{2 l+2+s}\left(8 \cdot f_{k}^{\prime} \cdot f^{m} \cdot \widetilde{q}_{l}\left(f^{2}\right)\right) \leq 2 l+1+2^{a(l)}\left(2^{s}-2\right)
\end{gathered}
$$

for all $0 \leq l \leq\left\lfloor\frac{n}{2}\right\rfloor-1, s \geq 1$. Then there exist unique $a_{l} \in\{0,1\}$ for $0 \leq l \leq\left\lfloor\frac{n}{2}\right\rfloor-1$ such that

$$
\widetilde{q}_{n}:=q_{n}+\sum_{l=0}^{\left\lfloor\frac{n}{2}\right\rfloor-1} a_{l} \cdot 2^{2(n-l)-1} \cdot \widetilde{q}_{l}
$$

satisfies

$$
8 \cdot f_{k}^{\prime} \cdot f^{m} \cdot \tilde{q}_{n}\left(f^{2}\right) \in 4 \cdot \mathbb{Z}[\chi] / I\langle 2 n+2\rangle .
$$

Moreover, we have

$$
\begin{aligned}
c_{2 n+3}\left(8 \cdot f_{k}^{\prime} \cdot f^{m} \cdot \tilde{q}_{n}\left(f^{2}\right)\right) & =2 n+1, \\
c_{2 n+2+s}\left(8 \cdot f_{k}^{\prime} \cdot f^{m} \cdot \tilde{q}_{n}\left(f^{2}\right)\right) & \leq 2 n+1+2^{a(n)}\left(2^{s}-2\right) \text { for all } s \geq 1 .
\end{aligned}
$$


Remark 5.25 We note that the statement (5-11) is proved using only the assumptions (5-8) and (5-9). The proof of the statement (5-12) on the other hand uses the assumptions (5-8), (5-9) and (5-10) for $s=2$. Since we are using induction we need to prove the statement (5-13) for $s=2$, but the proof of that uses (5-10) for $s=3$ and so on. This is the reason we need to prove the statements (5-13) for all $s \geq 1$.

Proof of Proposition 5.24 The proof proceeds in 3 steps.

(A) (Proof of (5-11), existence) From Proposition 5.22 we know that $b(n)=0$ implies

$$
8 \cdot f_{k}^{\prime} \cdot f^{m} \cdot q_{n}\left(f^{2}\right) \in 4 \cdot \mathbb{Z}[\chi] / I\langle 2 n+2\rangle,
$$

so we can set $a_{l}:=0$ for $0 \leq l \leq\left\lfloor\frac{n}{2}\right\rfloor-1$ if $b(n)=0$.

In the case $b(n)>0$ we have

$$
8 \cdot f_{k}^{\prime} \cdot f^{m} \cdot q_{n}\left(f^{2}\right) \cdot(1-\chi)^{2 b(n)-1} \in 4 \cdot \mathbb{Z}[\chi] / I\langle 2 n+2\rangle .
$$

Let $c \geq 0$ be the smallest number such that there exist coefficients $a_{l}$ satisfying

$(5-14) 8 \cdot f_{k}^{\prime} \cdot f^{m} \cdot\left(q_{n}\left(f^{2}\right)+\sum_{l=0}^{\left\lfloor\frac{n}{2}\right\rfloor-1} a_{l} \cdot 2^{2(n-l)-1} \cdot \widetilde{q}_{l}\left(f^{2}\right)\right) \cdot(1-\chi)^{c} \in 4 \cdot \mathbb{Z}[\chi] / I\langle 2 n+2\rangle$.

We have to show $c=0$. We will give a proof by contradiction and assume that $c>0$. We already know that

$$
c \leq 2 b(n)-1 \leq b(n)+2^{a(n)}-2=n-1 .
$$

Let $\left(a_{l}\right)$ be a choice of coefficients with the property (5-14). We set

$$
g_{1}(\chi):=8 \cdot f_{k}^{\prime} \cdot f^{m} \cdot q_{n}\left(f^{2}\right)+8 \cdot f_{k}^{\prime} \cdot f^{m} \cdot \sum_{l=0}^{\left\lfloor\frac{n}{2}\right\rfloor-1} a_{l} \cdot 2^{2(n-l)-1} \cdot \widetilde{q}_{l}\left(f^{2}\right) .
$$

Notice that $g_{1}(\chi) \in 2 \cdot \mathbb{Z}[\chi] / I\langle 2 n+2\rangle$ because all summands lie in this ring (use Lemma 5.16 and (5-8) for the summands indexed by $l$ ). From Lemma 5.13 we conclude that $c$ is odd. We set $l^{\prime}:=\frac{c-1}{2} \leq\left\lfloor\frac{n}{2}\right\rfloor-1$. Define

$$
g_{2}(\chi):=8 \cdot f_{k}^{\prime} \cdot f^{m} \cdot(-1)^{a_{l^{\prime}}} \cdot 2^{2\left(n-l^{\prime}\right)-1} \cdot \widetilde{q}_{l^{\prime}}\left(f^{2}\right) .
$$

Using Lemma 5.16 and (5-8) for $l^{\prime}$ we get $g_{2}(\chi) \in 2 \cdot \mathbb{Z}[\chi] / I\langle 2 n+2\rangle$. Using Corollary 5.17 and (5-9) for $l^{\prime}$ we see $c_{2 n+2}\left(g_{2}\right)=c$. Now, we can use Lemma 5.14 and get

$$
\left(g_{1}(\chi)+g_{2}(\chi)\right) \cdot(1-\chi)^{c-1} \in 4 \cdot \mathbb{Z}[\chi] / I\langle K\rangle .
$$


But this means that

$8 \cdot f_{k}^{\prime} \cdot f^{m} \cdot\left(q_{n}\left(f^{2}\right)+\sum_{l=0}^{\left\lfloor\frac{n}{2}\right\rfloor-1} a_{l}^{\prime} \cdot 2^{2(n-l)-1} \cdot \widetilde{q}_{l}\left(f^{2}\right)\right) \cdot(1-\chi)^{c-1} \in 4 \cdot \mathbb{Z}[\chi] / I\langle 2 n+2\rangle$ where the coefficients $\left(a_{l}^{\prime}\right)$ are given by

$$
a_{l}^{\prime}:= \begin{cases}a_{l} & l \neq l^{\prime}, \\ a_{l^{\prime}}+(-1)^{a_{l^{\prime}}} & l=l^{\prime} .\end{cases}
$$

This is a contradiction to the minimality of $c$.

(B) (Proof of (5-11), uniqueness) Our next aim is to show the uniqueness of the coefficients. We will give a proof by contradiction. Assume that there exist two different choices of coefficients $\left(a_{l}\right),\left(a_{l}^{\prime}\right)$ such that the corresponding $\widetilde{q}_{n}, \widetilde{q}_{n}^{\prime}$ satisfy

$$
8 \cdot f_{k}^{\prime} \cdot f^{m} \cdot \tilde{q}_{n}\left(f^{2}\right), 8 \cdot f_{k}^{\prime} \cdot f^{m} \cdot \tilde{q}_{n}^{\prime}\left(f^{2}\right) \in 4 \cdot \mathbb{Z}[\chi] / I\langle 2 n+2\rangle .
$$

We set $b_{l}:=a_{l}-a_{l}^{\prime} \in\{-1,0,1\}$ and conclude

$$
8 \cdot f_{k}^{\prime} \cdot f^{m} \cdot\left(\sum_{l=0}^{\left\lfloor\frac{n}{2}\right\rfloor-1} b_{l} \cdot 2^{2(n-l)-1} \cdot \widetilde{q}_{l}\left(f^{2}\right)\right) \in 4 \cdot \mathbb{Z}[\chi] / I\langle 2 n+2\rangle .
$$

Let $\hat{l}$ be the largest element with $b_{\hat{l}} \neq 0$. Using Corollary 5.17 and (5-9), we conclude

$$
c_{2 n+2}\left(8 \cdot f_{k}^{\prime} \cdot f^{m} \cdot 2^{2(n-l)-1} \cdot \widetilde{q}_{l}\left(f^{2}\right)\right)=2 l+1 .
$$

Lemma 5.15 implies

$$
c_{2 n+2}\left(8 \cdot f_{k}^{\prime} \cdot f^{m} \cdot\left(\sum_{l=0}^{\left\lfloor\frac{n}{2}\right\rfloor-1} b_{l} \cdot 2^{2(n-l)-1} \cdot \widetilde{q}_{l}\left(f^{2}\right)\right)\right)=2 \hat{l}+1 .
$$

This is a contradiction to (5-15).

(C) (Proofs of (5-12) and (5-13)) It remains to prove

$$
\begin{aligned}
c_{2 n+3}\left(8 \cdot f_{k}^{\prime} \cdot f^{m} \cdot \tilde{q}_{n}\left(f^{2}\right)\right) & =2 n+1, \\
c_{2 n+2+s}\left(8 \cdot f_{k}^{\prime} \cdot f^{m} \cdot \tilde{q}_{n}\left(f^{2}\right)\right) & \leq 2 n+1+2^{a(n)}\left(2^{s}-2\right) \text { for all } s \geq 1 .
\end{aligned}
$$

Using Corollary 5.17 and (5-10) for any $l<n$ and for any $s^{\prime} \geq 1$ we obtain

$$
c_{2 n+1+s^{\prime}}\left(8 \cdot f_{k}^{\prime} \cdot f^{m} \cdot 2^{2(n-l)-1} \cdot \widetilde{q}_{l}\left(f^{2}\right)\right) \leq 2 l+1+2^{a(l)}\left(2^{s^{\prime}}-2\right) .
$$


If $l \leq\left\lfloor\frac{n}{2}\right\rfloor-1$ and $s^{\prime} \geq 2$, we set $s:=s^{\prime}-1$ and conclude

$$
c_{2 n+2+s}\left(8 \cdot f_{k}^{\prime} \cdot f^{m} \cdot 2^{2(n-l)-1} \cdot \widetilde{q}_{l}\left(f^{2}\right)\right) \leq 2 n+1+2^{a(n)}\left(2^{s}-2\right)
$$

because

$$
\begin{aligned}
2 l+1+2^{a(l)}\left(2^{s+1}-2\right) & \leq 2\left(\frac{n}{2}-1\right)+1+2^{a(n)-1}\left(2^{s+1}-2\right) \\
& =2 n+1+2^{a(n)}\left(2^{s}-2\right)-b(n) \leq 2 n+1+2^{a(n)}\left(2^{s}-2\right) .
\end{aligned}
$$

If $s^{\prime}=2$ we obtain even better

$$
c_{2 n+3}\left(8 \cdot f_{k}^{\prime} \cdot f^{m} \cdot 2^{2(n-l)-1} \cdot \widetilde{q}_{l}\left(f^{2}\right)\right) \leq 2 n
$$

because

$$
2 l+1+2^{a(l)}\left(2^{2}-2\right) \leq 2\left(\frac{n}{2}-1\right)+1+2^{a(n)}=2 n-b(n) \leq 2 n .
$$

Therefore, it suffices to show

$$
\begin{aligned}
c_{2 n+3}\left(8 \cdot f_{k}^{\prime} \cdot f^{m} \cdot q_{n}\left(f^{2}\right)\right) & =2 n+1, \\
c_{2 n+2+s}\left(8 \cdot f_{k}^{\prime} \cdot f^{m} \cdot q_{n}\left(f^{2}\right)\right) & \leq 2 n+1+2^{a(n)}\left(2^{s}-2\right) \text { for all } s \geq 1 .
\end{aligned}
$$

But this was proved in Proposition 5.22.

Notice that the assumptions in Proposition 5.24 are trivially fulfilled if $n=0,1$.

Definition 5.26 We define $r_{n}^{-} \in \mathbb{Z}[\chi]$ as the polynomials $\widetilde{q}_{n}$ we obtain successively from Proposition 5.24 starting with $n=0$ and proceeding with $n=1,2,3, \ldots$

For example, $r_{0}^{-}=q_{0}, r_{1}^{-}=q_{1}, r_{2}^{-}=q_{2}+2^{3} \cdot q_{0}, r_{3}^{-}=q_{3}, r_{4}^{-}=q_{4}+2^{7} \cdot q_{0}$.

Corollary 5.27 The polynomial $r_{n}^{-}$is of degree $n \in \mathbb{N}$ with leading coefficient 1 and satisfies

$$
\begin{aligned}
8 \cdot f_{k}^{\prime} \cdot f^{m} \cdot r_{n}^{-}\left(f^{2}\right) & \in 4 \cdot \mathbb{Z}[\chi] / I\langle 2 n+2\rangle, \\
c_{2 n+3}\left(8 \cdot f_{k}^{\prime} \cdot f^{m} \cdot r_{n}^{-}\left(f^{2}\right)\right) & =2 n+1 .
\end{aligned}
$$

\subsection{The equality $A_{K}^{k}(d)=B_{K}(d)$}

Are the polynomials $r_{n}^{-}$best possible? Or does there exist a polynomial $q$ of degree $n$ with leading coefficient 1 such that $8 \cdot f_{k} \cdot q\left(f^{2}\right) \in \mathbb{Z}[\chi] / I\langle 2 n+3\rangle$ ? In this subsection 
we will see that any polynomial $q$ of degree $n$ with the property $8 \cdot f_{k} \cdot q\left(f^{2}\right) \in$ $\mathbb{Z}[\chi] / I\langle 2 n+3\rangle$ is of the shape

$$
\sum_{l=0}^{n} a_{l} \cdot 2^{\max \{2(n-l)+1,0\}} \cdot r_{l}^{-}
$$

with $a_{l} \in \mathbb{Z}$. Hence such a polynomial can not have 1 as leading coefficient. We first prove $A_{K}^{k}(d)=B_{K}(d)$ for $d=2 e+1$. Recall that

$$
\begin{aligned}
& A_{K}^{k}(2 e+1):=\left\{q \in \mathbb{Z}[x] \mid \operatorname{deg}(q) \leq e-1,8 \cdot f_{k} \cdot q\left(f^{2}\right) \in 4 \cdot \mathbb{Z}[\chi] / I\langle K\rangle\right\}, \\
& B_{K}(2 e+1):=\left\{\sum_{n=0}^{e-1} a_{n} \cdot 2^{\max \{K-2 n-2,0\}} \cdot r_{n}^{-} \mid a_{n} \in \mathbb{Z}\right\} .
\end{aligned}
$$

We want to consider a slightly more general situation and prove $A_{K}^{k, m}(2 e+1)=$ $B_{K}(2 e+1)$ where $A_{K}^{k, m}(2 e+1)$ is defined as follows.

Definition 5.28 Let $K, k \geq 1, e \geq 2, m \in\{1,2\}$. Define

$$
A_{K}^{k, m}(2 e+1):=\left\{q \in \mathbb{Z}[x] \mid \operatorname{deg}(q) \leq e-1,8 \cdot f_{k}^{\prime} \cdot f^{m} \cdot q\left(f^{2}\right) \in 4 \cdot \mathbb{Z}[\chi] / I\langle K\rangle\right\} .
$$

Notice that $A_{K}^{k, 1}(2 e+1)=A_{K}^{k}(2 e+1)$.

Theorem 5.29 Let $K, k \geq 1, e \geq 2, m \in\{1,2\}$. Then $A_{K}^{k, m}(2 e+1)=B_{K}(2 e+1)$. In particular,

$$
A_{K}^{k}(2 e+1)=B_{K}(2 e+1) \text {. }
$$

Remark 5.30 We proceed by induction with respect to $K$. The main ingredients in the proof are Corollary 5.27, Lemma 5.16, resp. Corollary 5.17 and Lemma 5.15. The more interesting part is the inclusion $A_{K}^{k, m}(2 e+1) \subseteq B_{K}(2 e+1)$ where this time the $c_{K}$-technology is used as a tool to show that certain linear combinations of polynomials cannot be in $4 \cdot \mathbb{Z}[\chi] / I\langle K\rangle$.

Proof of Theorem 5.29 Since $8 \cdot f_{k}^{\prime} \cdot f^{m} \cdot r_{n}^{-}\left(f^{2}\right) \in 4 \cdot \mathbb{Z}[\chi] / I\langle 2 n+2\rangle$, Lemma 5.16 implies

$$
8 \cdot f_{k}^{\prime} \cdot f^{m} \cdot 2^{\max \{K-2 n-2,0\}} \cdot r_{n}^{-}\left(f^{2}\right) \in 4 \cdot \mathbb{Z}[\chi] / I\langle K\rangle .
$$

This proves $A_{K}^{k, m}(2 e+1) \supseteq B_{K}(2 e+1)$.

It remains to show $A_{K}^{k, m}(2 e+1) \subseteq B_{K}(2 e+1)$. We will give a proof by induction with respect to $K$. For the basis case $K=1$ we get

$$
A_{1}^{k, m}(2 e+1)=\{q \in \mathbb{Z}[x] \mid \operatorname{deg}(q) \leq e-1\}=B_{1}(2 e+1) .
$$


Inductive step: We assume that $A_{K-1}^{k, m}(2 e+1) \subseteq B_{K-1}(2 e+1)(K \geq 2)$ and have to prove $A_{K}^{k, m}(2 e+1) \subseteq B_{K}(2 e+1)$. Let $q \in A_{K}^{k, m}(2 e+1)$. Since

$$
A_{K}^{k, m}(2 e+1) \subseteq A_{K-1}^{k, m}(2 e+1) \subseteq B_{K-1}(2 e+1),
$$

we can write $q$ as $q=\sum_{n=0}^{e-1} a_{n} \cdot 2^{\max \{K-2 n-3,0\}} \cdot r_{n}^{-}$with $a_{n} \in \mathbb{Z}$. The polynomial $q$ lies in $B_{K}(2 e+1)$ if $a_{n}$ is even for all $n$ with $2 n+2 \leq K-1$. We set

$$
M:=\left\{0 \leq n \leq e-1 \mid 2 n+2 \leq K-1, a_{n} \text { is odd }\right\} .
$$

It remains to show $M=\varnothing$. We will give a proof by contradiction and assume $M \neq \varnothing$. Since $q \in A_{K}^{k, m}(2 e+1)$ and

$$
\begin{aligned}
& \sum_{n \notin M} a_{n} \cdot 2^{\max \{K-2 n-3,0\}} \cdot r_{n}^{-}+\sum_{n \in M}\left(a_{n}-1\right) \cdot 2^{\max \{K-2 n-3,0\}} \cdot r_{n}^{-} \\
& \in B_{K}(2 e+1) \subseteq A_{K}^{k, m}(2 e+1),
\end{aligned}
$$

we have

$$
\sum_{n \in M} 2^{K-2 n-3} \cdot r_{n}^{-} \in A_{K}^{k, m}(2 e+1) .
$$

This implies

$$
\sum_{n \in M} 8 \cdot f_{k}^{\prime} \cdot f^{m} \cdot 2^{K-2 n-3} \cdot r_{n}^{-}\left(f^{2}\right) \cdot(1-\chi)^{2 \cdot \max (M)} \in 4 \cdot \mathbb{Z}[\chi] / I\langle K\rangle .
$$

On the other hand, we conclude from Corollary 5.27 and Corollary 5.17 that

$$
c_{K}\left(8 \cdot f_{k}^{\prime} \cdot f^{m} \cdot 2^{K-2 n-3} \cdot r_{n}^{-}\left(f^{2}\right)\right)=2 n+1
$$

for all $n \in M$. Lemma 5.15 implies

$$
c_{K}\left(\sum_{n \in M} 8 \cdot f_{k}^{\prime} \cdot f^{m} \cdot 2^{K-2 n-3} \cdot r_{n}^{-}\left(f^{2}\right)\right)=2 \cdot \max (M)+1 .
$$

But this is a contradiction to (5-16).

We now come to the case $d=2 e$.

Definition 5.31 Define $\beta: \mathbb{Z}[x] \rightarrow \mathbb{Z}[x]$ by

and set

$$
\beta(q)(x):=\frac{x \cdot q(x)-q(1)}{x-1}
$$

$$
r_{n}^{+}:=\beta\left(r_{n}^{-}\right) \text {for } n \geq 0 .
$$


Notice that $\beta$ is an isomorphism of $\mathbb{Z}$-modules and preserves the degree of the polynomial. The inverse is given by

$$
\beta^{-1}(q)(x)=\frac{(x-1) \cdot q(x)+q(0)}{x} .
$$

$r_{n}^{+}$is a polynomial of degree $n$ with leading coefficient 1 .

Theorem 5.32 Let $K, k \geq 1, e \geq 3$. Then

$$
A_{K}^{k}(2 e)=B_{K}(2 e) \text {. }
$$

Proof Recall that

$$
\begin{aligned}
& A_{K}^{k}(2 e):=\left\{q \in \mathbb{Z}[x] \mid \operatorname{deg}(q) \leq e-2,8 \cdot f_{k}^{\prime} \cdot\left(f^{2}-1\right) \cdot q\left(f^{2}\right) \in 4 \cdot \mathbb{Z}[\chi] / I\langle K\rangle\right\}, \\
& B_{K}(2 e):=\left\{\sum_{n=0}^{e-2} a_{n} \cdot 2^{\max \{K-2 n-2,0\}} \cdot r_{n}^{+} \mid a_{n} \in \mathbb{Z}\right\} .
\end{aligned}
$$

For $q \in \mathbb{Z}[x]$ with $\operatorname{deg}(q) \leq e-2$ we conclude

$$
\begin{aligned}
q \in A_{K}^{k}(2 e) & \Longleftrightarrow 8 \cdot f_{k}^{\prime} \cdot\left(f^{2}-1\right) \cdot q\left(f^{2}\right) \in 4 \cdot \mathbb{Z}[\chi] / I\langle K\rangle \\
& \left.\Longleftrightarrow 8 \cdot f_{k}^{\prime} \cdot\left(\left(f^{2}-1\right) \cdot q\left(f^{2}\right)+q(0)\right) \in 4 \cdot \mathbb{Z}[\chi] / I\langle K\rangle \quad \text { (since } f_{k}^{\prime} \in R_{\widehat{G}}\right) \\
& \Longleftrightarrow 8 \cdot f_{k}^{\prime} \cdot f^{2} \cdot \beta^{-1}(q)\left(f^{2}\right) \in 4 \cdot \mathbb{Z}[\chi] / I\langle K\rangle \\
& \Longleftrightarrow \beta^{-1}(q) \in A_{K}^{k, 2}(2 e-1) \\
& \Longleftrightarrow \beta^{-1}(q) \in B_{K}(2 e-1) \quad \text { (see Theorem 5.29) } \\
& \Longleftrightarrow q \in B_{K}(2 e) .
\end{aligned}
$$

This completes the proof.

\section{References}

[1] MF Atiyah, I M Singer, The index of elliptic operators. III, Ann. of Math. (2) 87 (1968) 546-604 MR0236952

[2] P E Conner, E E Floyd, Differentiable periodic maps, Ergebnisse der Math. und ihrer Grenzgebiete 33, Academic Press, New York (1964) MR0176478

[3] I Hambleton, L R Taylor, A guide to the calculation of the surgery obstruction groups for finite groups, from: "Surveys on surgery theory, Vol. 1", (S Cappell, A Ranicki, J Rosenberg, editors), Ann. of Math. Stud. 145, Princeton Univ. Press (2000) 225-274 MR1747537 
[4] W Lück, A basic introduction to surgery theory, from: "Topology of high-dimensional manifolds, No. 1, 2 (Trieste, 2001)", (F T Farrell, L Göttsche, W Lück, editors), ICTP Lect. Notes 9, Abdus Salam Int. Cent. Theoret. Phys., Trieste (2002) 1-224 MR1937016

[5] T Macko, C Wegner, On the classification of fake lens spaces arXiv:0810.1196

[6] I Madsen, R J Milgram, The classifying spaces for surgery and cobordism of manifolds, Annals of Math. Studies 92, Princeton University Press (1979) MR548575

[7] S López de Medrano, Involutions on manifolds, Ergebnisse der Math. und ihrer Grenzgebiete 59, Springer, New York (1971) MR0298698

[8] J Milnor, Whitehead torsion, Bull. Amer. Math. Soc. 72 (1966) 358-426 MR0196736

[9] J W Morgan, D P Sullivan, The transversality characteristic class and linking cycles in surgery theory, Ann. of Math. (2) 99 (1974) 463-544 MR0350748

[10] T Petrie, The Atiyah-Singer invariant, the Wall groups $L_{n}(\pi, 1)$, and the function $\left(t e^{x}+1\right) /\left(t e^{x}-1\right)$, Ann. of Math. (2) 92 (1970) 174-187 MR0319216

[11] AA Ranicki, A composition formula for manifold structures arXiv: math.AT.0608705

[12] A A Ranicki, Algebraic L-theory and topological manifolds, Cambridge Tracts in Math. 102, Cambridge Univ. Press (1992) MR1211640

[13] C T C Wall, Surgery on compact manifolds, second edition, Math. Surveys and Monogr. 69, Amer. Math. Soc. (1999) MR1687388 Edited and with a foreword by A A Ranicki

[14] C M Young, Normal invariants of lens spaces, Canad. Math. Bull. 41 (1998) 374-384 MR1637689

TM, CW: Mathematisches Institut, Universität Münster Einsteinstraße 62, Münster D-48149, Germany

TM: Matematický Ústav SAV

Štefánikova 49, Bratislava SK-81473, Slovakia

macko@math.uni-muenster.de, c.wegner@uni-muenster.de

http://www.math.uni-muenster.de/u/macko

Received: 25 August 2008 Revised: 9 July 2009 